\title{
Wilson-'t Hooft lines as transfer matrices
}

\author{
Kazunobu Maruyoshi, ${ }^{a}$ Toshihiro Ota ${ }^{b, c}$ and Junya Yagi ${ }^{d, e}$ \\ ${ }^{a}$ Faculty of Science and Technology, Seikei University, \\ 3-3-1 Kichijoji-Kitamachi, Musashino-shi, Tokyo 180-8633 Japan \\ ${ }^{b}$ Department of Physics, Osaka University, \\ Toyonaka, Osaka 560-0043 Japan \\ ${ }^{c}$ interdisciplinary Theoretical \& Mathematical Sciences Program (iTHEMS), RIKEN, \\ Wako, Saitama 351-0198 Japan \\ ${ }^{d}$ Perimeter Institute for Theoretical Physics, \\ Waterloo, ON, N2L 2Y5, Canada \\ e Yau Mathematical Sciences Center, Tsinghua University, \\ Beijing 100084, P.R. China \\ E-mail: maruyoshi@st.seikei.ac.jp, tota@het.phys.sci.osaka-u.ac.jp, \\ junyagi@tsinghua.edu.cn
}

ABstract: We establish a correspondence between a class of Wilson-'t Hooft lines in four-dimensional $\mathcal{N}=2$ supersymmetric gauge theories described by circular quivers and transfer matrices constructed from dynamical L-operators for trigonometric quantum integrable systems. We compute the vacuum expectation values of the Wilson-'t Hooft lines in a twisted product space $S^{1} \times{ }_{\epsilon} \mathbb{R}^{2} \times \mathbb{R}$ by supersymmetric localization and show that they are equal to the Wigner transforms of the transfer matrices. A variant of the AGT correspondence implies an identification of the transfer matrices with Verlinde operators in Toda theory, which we also verify. We explain how these field theory setups are related to four-dimensional Chern-Simons theory via embedding into string theory and dualities.

Keywords: Brane Dynamics in Gauge Theories, Lattice Integrable Models, Supersymmetric Gauge Theory

ArXiv ePrint: 2009.12391 


\section{Contents}

1 Introduction 1

2 Transfer matrices for Wilson-'t Hooft lines 3

2.1 L-operators and quantum integrable systems 3

$\begin{array}{ll}2.2 \text { Elliptic L-operator } & 7\end{array}$

2.3 Trigonometric L-operators 8

3 Wilson-'t Hooft lines as transfer matrices 11

3.1 Wilson-'t Hooft lines in $S^{1} \times_{\epsilon} \mathbb{R}^{2} \times \mathbb{R} \quad 11$

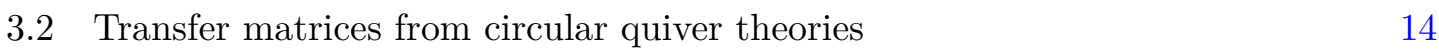

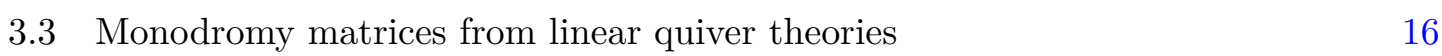

$\begin{array}{lll}3.4 & \text { Other representations } & 17\end{array}$

4 Transfer matrices from Verlinde operators 18

4.1 Verlinde operators and Wilson-'t Hooft lines 18

$\begin{array}{lll}4.2 & \text { Verlinde operators on a punctured torus } & 19\end{array}$

5 Brane realization $\quad 23$

\section{Introduction}

Supersymmetric gauge theories in four dimensions have various interrelated connections to quantum integrable systems. One such connection involves a family of surface defects in a class of $\mathcal{N}=1$ supersymmetric gauge theories, described by planer quivers. These surface defects act on the supersymmetric indices of the theories as commuting difference operators shifting flavor fugacities. It turns out that the difference operators coincide with transfer matrices of elliptic quantum integrable systems [1-3].

In this paper we present a similar correspondence. This correspondence, however, is between line defects in $\mathcal{N}=2$ supersymmetric gauge theories, described by circular quivers, and transfer matrices of trigonometric quantum integrable systems.

$\mathcal{N}=2$ supersymmetric gauge theories possess Wilson-'t Hooft lines which preserve half of the eight supercharges and carry both electric and magnetic charges [4]. The line defects that appear on the gauge theory side of the correspondence are such dyonic Wilson't Hooft lines. Roughly speaking, the statement of the correspondence is that the vacuum expectation values (vevs) of certain Wilson-'t Hooft lines are equal to classical values of transfer matrices of certain quantum integrable systems.

A more precise statement is as follows. Consider the theory described by an $n$-node circular quiver whose gauge group is the product of $n$ copies of $\mathrm{SU}(N)$. Let $m^{1}, \ldots, m^{n}$ 
be the mass parameters of the $n$ bifundamental hypermultiplets. We place the theory on $S^{1} \times_{\epsilon} \mathbb{R}^{2} \times \mathbb{R}$ and wind a Wilson-'t Hooft line $T_{\square, \sigma}$ around $S^{1}$, where $\epsilon$ is a twist parameter. The line operator $T_{\square, \sigma}$ is magnetically charged uniformly under the $\mathrm{SU}(N)$ factors; it transforms in the vector representation of the Langlands dual of each $\mathrm{SU}(N)$. The electric charge is specified by an $n$-tuple of signs $\sigma=\left(\sigma^{1}, \ldots, \sigma^{n}\right)$. On the Coulomb branch of vacua, the vev $\left\langle T_{\square, \sigma}\right\rangle$ of $T_{\square, \sigma}$ can be expressed in terms of trigonometric functions of the vevs of some vector multiplet fields. With respect to an appropriate holomorphic symplectic structure, the Weyl quantization of $\left\langle T_{\square, \sigma}\right\rangle$ gives a transfer matrix $\mathcal{T}_{\sigma, m}$ of a quantum integrable system, constructed from $n$ L-operators $\mathcal{L}_{\sigma^{1}, m^{1}}, \ldots, \mathcal{L}_{\sigma^{n}, m^{n}}$.

In section 2 , we introduce the L-operators $\mathcal{L}_{ \pm, m}$, which are the basic ingredients of the integrable system side, as particular trigonometric limits of the elliptic L-operator found in [5]. The elliptic L-operator satisfies the RLL relation with the elliptic dynamical R-matrix [6-8]. Consequently, its transfer matrix defines a quantum integrable system according to the standard procedure, as we review in this section. The key result of this section is the expression (2.43) for the Wigner transform $\left\langle\mathcal{T}_{\sigma, m}\right\rangle$ of the transfer matrix $\mathcal{T}_{\sigma, m}$, which is the inverse of the Weyl quantization $\left\langle\mathcal{T}_{\sigma, m}\right\rangle \mapsto \mathcal{T}_{\sigma, m}$.

We will establish the correspondence by supersymmetric localization [9], a technique to exactly compute the path integral for supersymmetric observables in theories with sufficiently large supersymmetry, placed on specific spacetime geometries. In the present setup, the relevant computation was carried out by Ito, Okuda and Taki [10].

In section 3, we apply the formula obtained in [10] to the Wilson-'t Hooft line $T_{\square, \sigma}$ and show that its vev reproduces the Wigner transform of the transfer matrix $\mathcal{T}_{\sigma, m}$ :

$$
\left\langle T_{\square, \sigma}\right\rangle=\left\langle\mathcal{T}_{\sigma, m}\right\rangle
$$

This is the main result of the paper. We will also explain how to recover L-operators and monodromy matrices from theories described by linear quivers. Moreover, we will propose a generalization of the correspondence to a broader class of Wilson-'t Hooft lines, in which the vector representation $\square$ is replaced by other representations.

Another route to compute the Wilson-'t Hooft line vev is via the AGT correspondence $[11,12]$. For a large class of $\mathcal{N}=2$ supersymmetric field theories, which includes the circular quiver theory, the AGT correspondence states that the partition function on an ellipsoid is equal to a correlation function in a two-dimensional conformal field theory (CFT), namely Toda theory. Under this correspondence, Wilson-'t Hooft lines in the former are mapped to line defects in the latter [13-15]. These line defects are known as Verlinde operators [16].

In section 4, we give an alternative derivation of the correspondence between Wilson't Hooft lines and transfer matrices that utilizes a variant of the AGT correspondence, proposed in [10], relating Verlinde operators to Wilson-'t Hooft lines in $S^{1} \times \mathbb{R}^{2} \times \mathbb{R}$, rather than in an ellipsoid. We consider the Verlinde operator corresponding to the Wilson't Hooft line in question, and verify that its action on conformal blocks matches the action of the transfer matrix in the quantum integrable system. 
While the correspondence between Wilson-'t Hooft lines and transfer matrices can be established by comparison of concrete calculations, mere matching does not explain why the correspondence exists in the first place.

In section 5, we provide an explanation using string theory. We will realize the circular quiver theory and the Wilson-'t Hooft line by branes, and apply string dualities to map the brane configuration to another one that realizes Costello's four-dimensional Chern-Simons theory $[17,18]$ and line defects in it. Four-dimensional Chern-Simons theory depends topologically on two directions, which form a cylinder in our case, and holomorphically on the remaining two directions. From this property it follows that line defects extending in the periodic topological direction produce transfer matrices of quantum integrable systems.

The embedding into string theory puts the correspondence treated in this paper in a bigger context. As discussed in [19], by brane realization and dualities, four-dimensional Chern-Simons theory is related to other field theory setups in which the same kind of integrability was found to arise. In particular, there is a duality frame that realizes the trigonometric limit of the setup of [2,3], thus connecting to the correspondence mentioned at the beginning.

\section{Transfer matrices for Wilson-'t Hooft lines}

In this section we discuss the integrable system side of the correspondence. After reviewing L-operators, transfer matrices and their relation to quantum integrable systems, we introduce an L-operator for the elliptic dynamical R-matrix. Then we define fundamental trigonometric L-operators as certain limits of the elliptic L-operator. These fundamental L-operators are building blocks of transfer matrices that correspond to Wilson-'t Hooft lines in $\mathcal{N}=2$ supersymmetric circular quiver theories.

\subsection{L-operators and quantum integrable systems}

Let $\mathfrak{h}$ be a finite-dimensional commutative complex Lie algebra and $V$ a finite-dimensional diagonalizable $\mathfrak{h}$-module. Choosing a basis $\left\{v_{i}\right\}$ of $V$ that is homogeneous with respect to weight decomposition, we denote the weight of $v_{i}$ by $h_{i}$ and the $(i, j)$ th entry of a matrix $M \in \operatorname{End}(V)$ by $M_{j}^{i}$. We write $\mathcal{M}_{\mathfrak{h}^{*}}$ for the field of meromorphic functions on the dual space $\mathfrak{h}^{*}$ of $\mathfrak{h}$.

Let $R: \mathbb{C} \times \mathfrak{h}^{*} \rightarrow \operatorname{End}(V \otimes V)$ be an $\operatorname{End}(V \otimes V)$-valued meromorphic function on $\mathbb{C} \times \mathfrak{h}^{*}$ that is invertible at a generic point $(z, a) \in \mathbb{C} \times \mathfrak{h}^{*}$. The coordinate $z$ is called the spectral parameter and $a$ is called the dynamical parameter.

In the discussions that follow, fundamental roles will be played by L-operators. By an $L$-operator for $R$, we mean a map $L: \mathbb{C} \rightarrow \operatorname{End}\left(V \otimes \mathcal{M}_{\mathfrak{h}^{*}} \otimes \mathcal{M}_{\mathfrak{h}^{*}}\right)$, which we think of as a matrix whose entries are linear operators on meromorphic functions on $\mathfrak{h}^{*} \times \mathfrak{h}^{*}{ }^{1}$ It must satisfy two conditions.

\footnotetext{
${ }^{1}$ Our definition of L-operators is more general than the one given in [22] in that L-operators may depend on two independent dynamical parameters. This generalization is necessary in order to treat the elliptic L-operator appearing in [5] in the formalism of dynamical R-matrix.
} 
First, its matrix elements act on $f \in \mathcal{M}_{\mathfrak{h}^{*}} \otimes \mathcal{M}_{\mathfrak{h}^{*}}$ as

$$
L(z)_{i}^{j} f\left(a^{1}, a^{2}\right)=L\left(z ; a^{1}, a^{2}\right)_{i}^{j} \Delta_{i}^{1} \Delta_{j}^{2} f\left(a^{1}, a^{2}\right),
$$

where $L\left(z ; a^{1}, a^{2}\right)_{i}^{j}$ is a meromorphic function on $\mathbb{C} \times \mathfrak{h}^{*} \times \mathfrak{h}^{*}$ and $\Delta_{i}^{1}, \Delta_{j}^{2}$ are difference operators such that

$$
\Delta_{i}^{1} f\left(a^{1}, a^{2}\right)=f\left(a^{1}-\epsilon h_{i}, a^{2}\right), \quad \Delta_{j}^{2} f\left(a^{1}, a^{2}\right)=f\left(a^{1}, a^{2}-\epsilon h_{j}\right) .
$$

Here $\epsilon$ is a fixed complex parameter.

Second, the L-operator satisfies the $R L L$ relation

$$
\begin{aligned}
\sum_{k, l} R\left(z-z^{\prime}, a^{2}\right)_{k l}^{m n} L\left(z ; a^{1}, a^{2}\right)_{i}^{k} L\left(z^{\prime} ; a^{1}-\epsilon h_{i}, a^{2}-\epsilon h_{k}\right)_{j}^{l} \\
=\sum_{k, l} L\left(z^{\prime} ; a^{1}, a^{2}\right)_{l}^{n} L\left(z ; a^{1}-\epsilon h_{l}, a^{2}-\epsilon h_{n}\right)_{k}^{m} R\left(z-z^{\prime}, a^{1}\right)_{i j}^{k l} .
\end{aligned}
$$

Equivalently, the operator relation

$$
\sum_{k, l} R\left(z-z^{\prime}, a^{2}\right)_{k l}^{m n} L(z)_{i}^{k} L\left(z^{\prime}\right)_{j}^{l}=\sum_{k, l} R\left(z-z^{\prime}, a^{1}\right)_{i j}^{k l} L\left(z^{\prime}\right)_{l}^{n} L(z)_{k}^{m}
$$

holds on any meromorphic function $f\left(a^{1}, a^{2}\right)$.

It is helpful, and will turn out to be physically meaningful, to represent the L-operator graphically as two crossing oriented line segments:

$$
L(z)=z \longrightarrow \Uparrow .
$$

The solid line extending in the horizontal direction has a spectral parameter. The graphical representation of a matrix element of the L-operator is

$$
L\left(z ; a^{1}, a^{2}\right)_{i}^{j}=z \underset{a^{1}-\epsilon h_{i}}{\stackrel{a^{1}}{(i)}} \underset{a^{2}-\epsilon h_{j}}{\stackrel{a^{2}}{\longrightarrow}} .
$$

Each edge of a solid line carries a state in $V$, and the state may change when the line crosses another line. To each region separated by lines, a dynamical parameter is assigned. The values of dynamical parameters on the two sides of a solid line carrying state $v_{i}$ differ by $\epsilon h_{i}$.

We also represent the operator $R$ as two crossing solid lines:

$$
R\left(z-z^{\prime}, a\right)_{i j}^{k l}=z-i \overbrace{z^{\prime}}^{a}(\hat{i}) \rightarrow
$$


Then, the RLL relation (2.3) simply means an equality between two configurations involving two solid and one double lines:

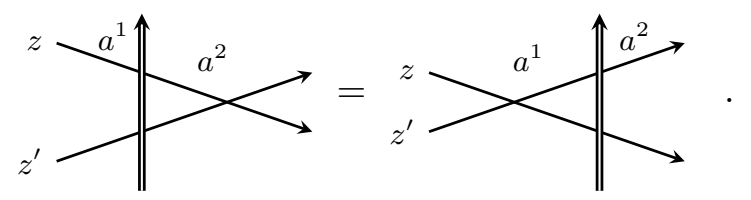

The states carried by the internal solid edges are summed over.

By comparing the values of the dynamical parameter assigned to the lower right regions of the two sides, we see that for $R$ to satisfy the RLL relation with some L-operators, generally it must commute with $h \otimes 1+1 \otimes h$ for all $h \in \mathfrak{h}$; in other words, $R(z, a)_{i j}^{k l}=0$ unless $h_{i}+h_{j}=h_{k}+h_{l}$. This is a consistency condition for the rule that determines how dynamical parameters change across solid lines.

Associated with an L-operator, there is an integrable quantum mechanical system consisting of particles moving in the space $\mathfrak{h}^{*}$. The Hilbert space of each particle is $\mathcal{M}_{\mathfrak{h}}$. (This is quantum mechanics in which real variables are analytically continued to complex ones.) The Hilbert space of the system is $\mathcal{M}_{\mathfrak{h}^{*}}^{\otimes n}$ if $n$ is the number of particles.

To construct this system, define the monodromy matrix $M: \mathbb{C} \rightarrow \operatorname{End}\left(V \otimes \mathcal{M}_{\mathfrak{h}^{*}}^{\otimes n+1}\right)$ by the product of $n$ copies of the L-operator: its matrix elements are given by

$$
M(z)_{i^{1}}^{i^{n+1}}=\sum_{i^{2}, \ldots, i^{n}} \prod_{r=1}^{n} L\left(z ; a^{r}, a^{r+1}\right)_{i^{r}}^{i^{r+1}} \prod_{s=1}^{n+1} \Delta_{i^{s}}^{s},
$$

acting on any meromorphic function $f\left(a^{1}, \ldots, a^{n+1}\right)$. (The superscript on $\Delta_{i}$ specifies the variable on which the difference operator acts.) This is a solid line crossing $n$ double lines:

$$
M(z)=z \longrightarrow \Uparrow \Uparrow \Uparrow
$$

Identifying $a^{n+1}=a^{1}$ and taking the trace, one obtains the transfer matrix $T: \mathbb{C} \rightarrow$ $\operatorname{End}\left(\mathcal{M}_{\mathfrak{h}^{*}}^{\otimes n}\right)$ :

$$
T(z)=\sum_{i^{1}, \ldots, i^{n}} \prod_{r=1}^{n} L\left(z ; a^{r}, a^{r+1}\right)_{i^{r}}^{i^{r+1}} \prod_{s=1}^{n} \Delta_{i^{s}}^{s}, \quad i^{n+1}=i^{1} .
$$

Graphically, $T(z)$ is represented by the same picture as above but with the horizontal direction made periodic.

By construction, $T$ is an $\operatorname{End}\left(\mathcal{M}_{\mathfrak{h}^{*}}^{\otimes n}\right)$-valued meromorphic function. As such, each coefficient $T_{m}$ in the Laurent expansion $T(z)=\sum_{m \in \mathbb{Z}} T_{m} z^{m}$ is an operator acting on the Hilbert space $\mathcal{M}_{\mathfrak{h}^{*}}^{\otimes n}$. Then, one may pick a particular linear combination of these coefficients and declare that it is the Hamiltonian of the quantum mechanical system. The Hamiltonian thus obtained is a difference operator, which is typical of relativistic systems.

Alternatively, one may think of this system as a one-dimensional periodic quantum spin chain. This spin chain is constructed from $n$ double lines extending in the longitudinal direction of a cylinder, as shown in figure 1(a). The dynamical parameter $a^{r}$ resides in the 


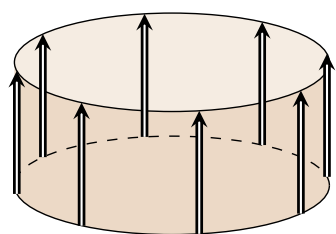

(a)

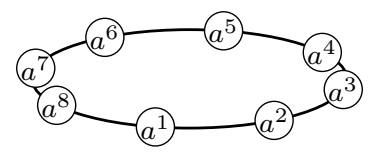

(b)

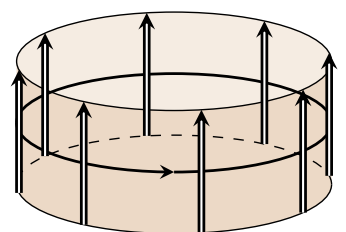

(c)

Figure 1. (a) Double lines in the longitudinal direction of a cylinder. (b) The corresponding quantum spin chain with continuous spin variables. (c) A solid line winding around the cylinder acts on the spin chain by the transfer matrix.

region sandwiched by the $r$ th and the $(r+1)$ th double lines. One regards the $n$ dynamical parameters $a^{1}, \ldots, a^{n}$ as continuous spin variables; see figure $1(\mathrm{~b})$. Thinking of the longitudinal direction as the time direction, the Hilbert space of the spin chain is again $\mathcal{M}_{\mathfrak{h}^{*}}^{\otimes n}$. An action of $T(z)$ on the Hilbert space is induced by an insertion of a solid line with spectral parameter $z$ in the circumferential direction of the cylinder, as in figure 1(c).

The integrability of the system is a consequence of the RLL relation. By repeated use of the RLL relation, one deduces that the monodromy matrix satisfies a similar relation:

$$
\sum_{k, l} R\left(z-z^{\prime}, a^{n+1}\right)_{k l}^{m n} M(z)_{i}^{k} M\left(z^{\prime}\right)_{j}^{l}=\sum_{k, l} R\left(z-z^{\prime}, a^{1}\right)_{i j}^{k l} M\left(z^{\prime}\right)_{l}^{n} M(z)_{k}^{m} .
$$

Multiplying both sides by $R^{-1}\left(z-z^{\prime}, a^{1}\right)_{m n}^{i j}$, setting $a^{n+1}=a^{1}$ and summing over $i, j, m$, $n$, one finds

$$
T(z) T\left(z^{\prime}\right)=T\left(z^{\prime}\right) T(z) .
$$

In other words, transfer matrices at different values of the spectral parameter commute. It follows that the Laurent coefficients $\left\{T_{m}\right\}$ mutually commute and, in particular, commute with the Hamiltonian. Hence, the system has a series of commuting conserved charges.

There is a slight generalization of the above construction of commuting transfer matrices. Suppose that $g \in \operatorname{End}(V)$ satisfies

$$
(g \otimes g) R(z, a)=R(z, a)(g \otimes g)
$$

and a subspace $W$ of $V$ is invariant under $R, R^{-1}, L$ and $g$. (For instance, the invariance of $W$ under $R$ means that $R(z, a)(W \otimes V) \subset W \otimes V$ and $R(z, a)(V \otimes W) \subset V \otimes W$ for all $z, a$.) Then, the trace can be twisted by $g$ and restricted to $W$ :

$$
T_{g, W}=\operatorname{Tr}_{W}(g M) .
$$

If $W_{1}, W_{2}$ are such invariant subspaces, then

$$
\left[T_{g, W_{1}}(z), T_{g, W_{2}}\left(z^{\prime}\right)\right]=0 .
$$

Thus, we get different kinds of transfer matrices labeled by invariant subspaces, and they commute with each other. A typical situation in which this construction applies is when 
$\mathfrak{h}$ is a Cartan subalgebra of a complex Lie algebra $\mathfrak{g}_{\mathbb{C}}, V$ is a direct sum of irreducible representations of $\mathfrak{g}_{\mathbb{C}}$, and $g$ is an element of $\mathfrak{g}_{\mathbb{C}}$.

Algebraically, L-operators give representations of dynamical quantum groups [20-22]. As an algebra, the dynamical quantum group corresponding to $R$ is generated by the meromorphic functions on $\mathbb{C} \times \mathfrak{h}^{*} \times \mathfrak{h}^{*}$, together with additional generators $l(z)_{j}^{i}, l^{-1}(z)_{j}^{i}$. The generators $l(z)_{j}^{i}$ are to be understood as the matrix elements of an abstract L-operator and satisfy the same relations as above; $l^{-1}(z)_{j}^{i}$ are the elements of the inverse matrix. This algebra has further structures (coproduct and counit) which make it an $\mathfrak{h}$-bialgebroid.

\section{$2.2 \quad$ Elliptic L-operator}

An important example of an L-operator is one for the elliptic dynamical R-matrix [6-8], which is a representation of the elliptic quantum group for $\mathfrak{s l}_{N}$. In this example, $\mathfrak{h}$ is the Cartan subalgebra of $\mathfrak{s l}_{N}$ and $V=\mathbb{C}^{N}$ is the vector representation of $\mathfrak{s l}_{N}$.

The Lie algebra $\mathfrak{s l}_{N}$ consists of the traceless complex $N \times N$ matrices and $\mathfrak{h}$ is the subalgebra of diagonal elements. We denote by $E_{i j} \in \mathfrak{g l}_{N}$ the matrix that has 1 in the $(i, j)$ th entry and 0 elsewhere, and by $E_{i j}^{*}$ the element of $\mathfrak{g l}_{N}^{*}=\operatorname{Hom}\left(\mathfrak{g l}_{N}, \mathbb{C}\right)$ such that $\left\langle E_{i j}, E_{k l}^{*}\right\rangle=\delta_{i k} \delta_{j l}$. (The bilinear map $\langle-,-\rangle: \mathfrak{g l}_{N} \times \mathfrak{g l}_{N}^{*} \rightarrow \mathbb{C}$ is the natural pairing.) The elements of $\mathfrak{h}$ are matrices of the form $\sum_{i=1}^{N} b_{i} E_{i i}$, with $\sum_{i=1}^{N} b_{i}=0$. Since $\mathfrak{h}$ is isomorphic to the quotient of the subspace of $\mathfrak{g l}_{N}$ consisting of the diagonal matrices by the subspace spanned by the identity matrix $I=\sum_{i=1}^{N} E_{i i}$, the dual space $\mathfrak{h}^{*}$ is isomorphic to the subspace of $\mathfrak{g l}_{N}^{*}$ consisting of elements of the form $\sum_{i=1}^{N} a_{i} E_{i i}^{*}$ such that $\left\langle I, \sum_{i=1}^{N} a_{i} E_{i i}^{*}\right\rangle=$ $\sum_{i=1}^{N} a_{i}=0$. Thus, $\mathfrak{h}^{*}$ may also be identified with the space of traceless diagonal matrices.

The natural action of $\mathfrak{s l}_{N}$ on $\mathbb{C}^{N}$ defines the vector representation of $\mathfrak{s l}_{N}$. In terms of the standard basis $\left\{e_{1}, \ldots, e_{N}\right\}$ of $\mathbb{C}^{N}$, we have $\sum_{j=1}^{N} a_{j} E_{j j} e_{i}=a_{i} e_{i}$. The weight of $e_{i}$ is therefore

$$
h_{i}=E_{i i}^{*}-\frac{1}{N} \sum_{j=1}^{N} E_{j j}^{*} .
$$

For $a \in \mathfrak{h}^{*}$, we write $a_{i}=\left\langle E_{i i}, a\right\rangle$. Then, $\sum_{i=1}^{N} a_{i}=0$ and $a=\sum_{i=1}^{N} a_{i} E_{i i}^{*}=\sum_{i=1}^{N} a_{i} h_{i}$.

Fix a point $\tau$ in the upper half plane, $\operatorname{Im} \tau>0$, and let

$$
\theta_{1}(z)=-\sum_{j \in \mathbb{Z}+\frac{1}{2}} e^{\pi \mathrm{i} j^{2} \tau+2 \pi \mathrm{i} j\left(z+\frac{1}{2}\right)}
$$

be Jacobi's first theta function. The elliptic dynamical $R$-matrix $R^{\mathrm{ell}}$ is defined by $[20-22]$

$$
R^{\mathrm{ell}}(z, a)=\sum_{i=1}^{N} E_{i i} \otimes E_{i i}+\sum_{i \neq j} \alpha\left(z, a_{i j}\right) E_{i i} \otimes E_{j j}+\sum_{i \neq j} \beta\left(z, a_{i j}\right) E_{j i} \otimes E_{i j}
$$

where $a_{i j}=a_{i}-a_{j}$ and

$$
\alpha(z, a)=\frac{\theta_{1}(a+\epsilon) \theta_{1}(-z)}{\theta_{1}(a) \theta_{1}(\epsilon-z)}, \quad \beta(z, a)=\frac{\theta_{1}(a-z) \theta_{1}(\epsilon)}{\theta_{1}(a) \theta_{1}(\epsilon-z)} .
$$


The elliptic L-operator $L^{\mathrm{ell}}$, which satisfies the RLL relation with $R^{\text {ell }}$, has the matrix elements given by [5]

$$
L_{w, y}^{\mathrm{ell}}\left(z ; a^{1}, a^{2}\right)_{i}^{j}=\frac{\theta_{1}\left(z-w+a_{j}^{2}-a_{i}^{1}\right)}{\theta_{1}(z-w)} \prod_{k(\neq i)} \frac{\theta_{1}\left(a_{k}^{1}-a_{j}^{2}-y\right)}{\theta_{1}\left(a_{k i}^{1}\right)} .
$$

The complex numbers $w, y$ may be thought of as spectral parameters for the corresponding double line. The presence of the two parameters is due to the fact that $R^{\text {ell }}(z, a)$ is invariant under shift of $a$ by a multiple of the identity matrix $I$ and in the RLL relation (2.3) the spectral parameters $z, z^{\prime}$ enter the R-matrix only through the difference $z-z^{\prime}$; note also that the L-operator can be multiplied by any function of the spectral parameter.

The elliptic dynamical R-matrix and the elliptic L-operator have many more properties than just that they satisfy the RLL relation. Most importantly, the R-matrix is a solution of the dynamical Yang-Baxter equation [20, 21, 23] and encodes the Boltzmann weights for a two-dimensional integrable lattice model [6-8]. This model is equivalent to the eight-vertex model [24, 25] (or more precisely, the Belavin model [26] which is an $\mathfrak{s l}_{N}$ generalization of the eight-vertex model) in the sense that the transfer matrices of the two models are related by a similarity transformation. The elliptic L-operator, on the other hand, satisfies the RLL relation with another R-matrix which describes an integrable lattice model called the Bazhanov-Sergeev model $[27,28]$, whose spins variables take values in $\mathfrak{h}^{*}$. We will not discuss these aspects in this paper. The interested reader is referred to [3] for more details.

\subsection{Trigonometric L-operators}

The L-operators that appear in the correspondence with Wilson-'t Hooft lines are obtained from the elliptic L-operator $L^{\text {ell }}$ via the trigonometric limit $\tau \rightarrow \mathrm{i} \infty$. For comparison with gauge theory results, we actually need to express these L-operators in somewhat different forms.

First, we describe L-operators in a quantum mechanical language. Let us explain this description in the case in which $\mathfrak{h}$ is the Cartan subalgebra of $\mathfrak{s l}_{N}$. Recall that $\mathfrak{s l}_{N}$ has simple coroots

$$
\alpha_{i}^{\vee}=E_{i i}-E_{i+1, i+1}, \quad i=1, \ldots, N-1,
$$

and the fundamental weights

$$
\omega_{i}=\left(\alpha_{i}^{\vee}\right)^{*}=\sum_{j=1}^{i} h_{j} .
$$

Consider quantum mechanics of a particle living in $\mathfrak{h}^{*} \times \mathfrak{h}^{*}$, with Planck constant

$$
\hbar=-\frac{\epsilon}{2 \pi} \text {. }
$$

If $\left(a^{1}, a^{2}\right) \in \mathfrak{h}^{*} \times \mathfrak{h}^{*}$ is the position of the particle, we write $a^{r}=\sum_{i=1}^{N-1} q_{i}^{r} \omega_{i}, r=1,2$. Similarly, we write the momenta $\left(b^{1}, b^{2}\right) \in \mathfrak{h} \times \mathfrak{h}$ of the particle as $b^{r}=\sum_{i=1}^{N-1} p_{i}^{r} \alpha_{i}^{\vee}$. The corresponding position and momentum operators $\hat{q}_{i}^{r}, \hat{p}_{i}^{s}$ satisfy the canonical commutation relations:

$$
\left[\hat{q}_{i}^{r}, \hat{p}_{j}^{s}\right]=\mathrm{i} \hbar \delta^{r s} \delta_{i j}, \quad i, j=1, \ldots, N-1 .
$$

(As before, we are treating $q_{i}^{r}, p_{i}^{r}$ as analytically continued variables.) 
To rewrite the commutation relations in a form that is invariant under the action of the Weyl group, we make a change of basis

$$
a^{r}=\sum_{i=1}^{N} a_{i}^{r} E_{i i}^{*}, \quad b^{r}=\sum_{i=1}^{N} b_{i}^{r} E_{i i} .
$$

Then, the corresponding observables $\hat{a}_{i}^{r}, \hat{b}_{i}^{r}$ obey the traceless condition, $\sum_{i=1}^{N} \hat{a}_{i}^{r}=$ $\sum_{i=1}^{N} \hat{b}_{i}^{r}=0$, and satisfy the commutation relations

$$
\left[\hat{a}_{i}^{r}, \hat{b}_{j}^{s}\right]=\mathrm{i} \hbar \delta^{r s}\left(\delta_{i j}-\frac{1}{N}\right), \quad i, j=1, \ldots, N .
$$

Using these observables we can identify the matrix elements of an L-operator $L$ with an operator in the Hilbert space of this quantum mechanical system:

$$
L(z)_{i}^{j}=L\left(z ; \hat{a}^{1}, \hat{a}^{2}\right)_{i}^{j} e^{2 \pi \mathrm{i}\left(\hat{b}_{i}^{1}+\hat{b}_{j}^{2}\right)} .
$$

In quantum mechanics, there is an invertible map from functions on the classical phase space to operators in the Hilbert space, known as the Weyl transform: if $q$ and $p$ are canonically conjugate variables, it maps

$$
f(q, p) \mapsto \hat{f}(\hat{q}, \hat{p})=\int_{\mathbb{R}^{4}} \mathrm{~d} x \mathrm{~d} y \mathrm{~d} p \mathrm{~d} q f(q, p) e^{\mathrm{i}(x(\hat{q}-q)+y(\hat{p}-p))} .
$$

The inverse map is the Wigner transform, which we denote by $\langle-\rangle$ :

$$
f(\hat{q}, \hat{p}) \mapsto\langle f(\hat{q}, \hat{p})\rangle=\int_{\mathbb{R}} \mathrm{d} x e^{\mathrm{i} p x / \hbar}\left\langle q+\frac{1}{2} x|\hat{f}(\hat{q}, \hat{p})| q-\frac{1}{2} x\right\rangle .
$$

In the situation at hand, if we rewrite the expression (2.28) as

$$
L(z)_{i}^{j}=e^{\pi \mathrm{i}\left(\hat{b}_{i}^{1}+\hat{b}_{j}^{2}\right)} \widetilde{L}\left(z ; \hat{a}^{1}, \hat{a}^{2}\right)_{i}^{j} e^{\pi \mathrm{i}\left(\hat{b}_{i}^{1}+\hat{b}_{j}^{2}\right)},
$$

then we have

$$
\left\langle L(z)_{i}^{j}\right\rangle=e^{2 \pi \mathrm{i}\left(b_{i}^{1}+b_{j}^{2}\right)} \widetilde{L}\left(z ; a^{1}, a^{2}\right)_{i}^{j} .
$$

Next, we apply a similarity transformation to the elliptic L-operator. Assume $\operatorname{Im} \epsilon>0$ and let

$$
\Gamma(z, \tau, \epsilon)=\prod_{m, n=0}^{\infty} \frac{1-e^{2 \pi \mathrm{i}((m+1) \tau+(n+1) \epsilon-z)}}{1-e^{2 \pi \mathrm{i}(m \tau+n \epsilon+z)}}
$$

be the elliptic gamma function. Then, $\bar{\Gamma}(z)=e^{\pi \mathrm{i} z^{2} / 2 \epsilon} \Gamma(z, \tau, \epsilon)$ has the property that $\bar{\Gamma}(z+\epsilon, \tau, \epsilon)=g(\tau, \epsilon) \theta_{1}(z) \bar{\Gamma}(z, \tau, \epsilon)$ for some function $g(\tau, \epsilon)$. We define the conjugated L-operator $\mathcal{L}_{w, m}^{\text {ell }}(z)$ by

$$
\mathcal{L}_{w, m}^{\mathrm{ell}}(z)_{i}^{j}=\Phi_{m-\frac{1}{2} \epsilon} L_{w, m-\frac{1}{2} \epsilon}^{\mathrm{ell}}(z)_{i}^{j} \Phi_{m-\frac{1}{2} \epsilon}^{-1},
$$

where

$$
\Phi_{y}=\prod_{k, l=1}^{N} \bar{\Gamma}\left(\hat{a}_{k}^{1}-\hat{a}_{l}^{2}-y\right)^{\frac{1}{2}} \prod_{k \neq l} \bar{\Gamma}\left(\hat{a}_{k l}^{1}\right)^{-\frac{1}{2}} .
$$


It has the Wigner transform

$$
\begin{aligned}
\left\langle\mathcal{L}_{w, m}^{\mathrm{ell}}(z)_{i}^{j}\right\rangle= & e^{2 \pi \mathrm{i}\left(b_{i}^{1}+b_{j}^{2}\right)} \frac{\theta_{1}\left(z-w+a_{j}^{2}-a_{i}^{1}\right)}{\theta_{1}(z-w)} \\
& \times\left(\frac{\prod_{k(\neq i)} \theta_{1}\left(a_{k}^{1}-a_{j}^{2}-m\right) \prod_{l(\neq j)} \theta_{1}\left(a_{i}^{1}-a_{l}^{2}-m\right)}{\prod_{k(\neq i)} \theta_{1}\left(a_{k i}^{1}-\frac{1}{2} \epsilon\right) \theta_{1}\left(a_{i k}^{1}-\frac{1}{2} \epsilon\right)}\right)^{\frac{1}{2}} .
\end{aligned}
$$

With these preparations, let us finally take the trigonometric limit to define the trigonometric L-operator:

$$
\mathcal{L}_{w, m}=\lim _{\tau \rightarrow \mathrm{i} \infty} \mathcal{L}_{w, m}^{\mathrm{ell}} .
$$

The trigonometric L-operator satisfies the RLL relation with the trigonometric limit $R^{\text {trig }}$. of the elliptic R-matrix $R^{\text {ell }}$. Concretely, $\mathcal{L}_{w, m}$ and $R^{\text {trig }}$ are obtained from $\mathcal{L}_{w, m}^{\text {ell }}$ and $R^{\text {ell }}$ by the replacement $\theta_{1}(z) \rightarrow \sin (\pi z)$.

Once we are in the trigonometric setup, the quasi-periodicity in $z \rightarrow z+\tau$ is lost and we can further take the limits $w \rightarrow \pm \mathrm{i} \infty$. This allows us to introduce more fundamental L-operators:

$$
\mathcal{L}_{ \pm, m}=\lim _{w \rightarrow \pm \mathrm{i} \infty} \mathcal{L}_{w, m}
$$

These L-operators do not depend on the spectral parameters $z, w$, and their matrix elements have the Wigner transforms

$$
\left\langle\left(\mathcal{L}_{ \pm, m}\right)_{i}^{j}\right\rangle=e^{2 \pi \mathrm{i}\left(b_{i}^{1}+b_{j}^{2}\right)} e^{ \pm \pi \mathrm{i}\left(a_{j}^{2}-a_{i}^{1}\right)} \ell_{m}\left(a^{1}, a^{2}\right)_{i}^{j},
$$

with

$$
\ell_{m}\left(a^{1}, a^{2}\right)_{i}^{j}=\left(\frac{\prod_{k(\neq i)} \sin \pi\left(a_{k}^{1}-a_{j}^{2}-m\right) \prod_{l(\neq j)} \sin \pi\left(a_{i}^{1}-a_{l}^{2}-m\right)}{\prod_{k(\neq i)} \sin \pi\left(a_{k i}^{1}-\frac{1}{2} \epsilon\right) \sin \pi\left(a_{i k}^{1}-\frac{1}{2} \epsilon\right)}\right)^{\frac{1}{2}} .
$$

The L-operator for arbitrary parameters $z, w$ can be realized as a linear combination of $\mathcal{L}_{ \pm, m}:$

$$
\mathcal{L}_{w, m}(z)=\frac{e^{\pi \mathrm{i}(z-w)} \mathcal{L}_{+, m}-e^{-\pi \mathrm{i}(z-w)} \mathcal{L}_{-, m}}{\sin \pi(z-w)} .
$$

The monodromy matrix $\mathcal{M}_{\sigma, m}$ constructed from $\mathcal{L}_{ \pm, m}$ is labeled by an $n$-tuple of signs $\sigma=\left(\sigma^{1}, \ldots, \sigma^{n}\right) \in\{ \pm\}^{n}$ and an $n$-tuple of complex numbers $m=\left(m^{1}, \ldots, m^{n}\right)$ :

$$
\left\langle\left(\mathcal{M}_{\sigma, m}\right)_{i^{1}}^{i^{n+1}}\right\rangle=\sum_{i^{2}, \ldots, i^{n}} \prod_{s=1}^{n+1} e^{2 \pi \mathrm{i} b_{i}^{s}} \prod_{r=1}^{n} e^{\sigma^{r} \pi \mathrm{i}\left(a_{i^{r+1}}^{r+1}-a_{i^{r}}^{r}\right)} \ell_{m^{r}}\left(a^{r}, a^{r+1}\right)_{i^{r}}^{i^{r+1}}
$$

The corresponding transfer matrix $\mathcal{T}_{\sigma, m}$ has the Wigner transform

$$
\left\langle\mathcal{T}_{\sigma, m}\right\rangle=\sum_{i^{1}, \ldots, i^{n}} \prod_{r=1}^{n} e^{2 \pi \mathrm{i} b_{i^{r}}^{r}} e^{\sigma^{r} \pi \mathrm{i}\left(a_{i^{r+1}}^{r+1}-a_{i^{r}}^{r}\right)} \ell_{m^{r}}\left(a^{r}, a^{r+1}\right)_{i^{r}}^{i^{r+1}},
$$

with $a^{n+1}=a^{1}, i^{n+1}=i^{1}$. Our claim is that these quantities equal the vevs of Wilson-'t Hooft lines in $\mathcal{N}=2$ supersymmetric gauge theories. 


\section{Wilson-'t Hooft lines as transfer matrices}

In the previous section we defined the fundamental trigonometric L-operators (2.38) and calculated transfer matrices constructed from them. As explained in section 1, these transfer matrices are expected to have interpretations as Wilson-'t Hooft lines in $\mathcal{N}=2$ supersymmetric gauge theories described by a circular quiver. In this section we verify this expectation by computing the vevs of the corresponding Wilson-'t Hooft lines.

\subsection{Wilson-'t Hooft lines in $S^{1} \times_{\epsilon} \mathbb{R}^{2} \times \mathbb{R}$}

Consider a four-dimensional gauge theory whose gauge group is a compact Lie group $G$ with Lie algebra $\mathfrak{g}$. Choosing a maximal torus $T \subset G$ with Lie algebra $\mathfrak{t}$, we let $\Lambda_{\mathrm{r}}(\mathfrak{g}) \subset \mathfrak{t}^{*}$ and $\Lambda_{\text {cr }}(\mathfrak{g}) \subset \mathfrak{t}$ be the root lattice and the coroot lattice of $\mathfrak{g}$, respectively. Their duals are the coweight lattice $\Lambda_{\mathrm{cw}}(\mathfrak{g})=\Lambda_{\mathrm{r}}(\mathfrak{g})^{\vee} \subset \mathfrak{t}$ and the weight lattice $\Lambda_{\mathrm{w}}(\mathfrak{g})=\Lambda_{\mathrm{cr}}(\mathfrak{g})^{\vee} \subset \mathfrak{t}^{*}$.

An 't Hooft line is the worldline of a very heavy monopole, that is, a nondynamical magnetically charged particle. In the presence of an 't Hooft line, the gauge field of the theory has a singularity at the location of the monopole: in terms of the polar angle $\theta$ and the azimuthal angle $\phi$ of the spherical coordinates centered at the monopole, the gauge field behaves as

$$
A=\frac{\mathbf{m}}{2}(1-\cos \theta) \mathrm{d} \phi+\cdots,
$$

where $\cdots$ represents less singular terms. (For simplicity we are setting the gauge theory theta-angles to zero.) The coefficient $\mathbf{m}$ is the magnetic charge of the monopole. Different singular gauge field configurations of the above form describe the same monopole if their magnetic charges are related by gauge transformation. It follows that $\mathbf{m}$ can be chosen from $\mathfrak{t}$, and the choice is meaningful only up to the action of the Weyl group $W(G)$ of $G$.

The above expression of $A$ is valid in a trivialization over a coordinate patch that contains the point $\theta=0$ of a two-sphere surrounding the monopole. At $\theta=\pi$, there is a "Dirac string" which supports an unphysical magnetic flux. For the Dirac string to be invisible (or more precisely, for the gauge transformation by $\exp (\operatorname{im} \phi)$ which allows us to go to the coordinate patch containing $\theta=\pi$ to be well defined), we must have

$$
\langle\mathbf{m}, w\rangle \in \mathbb{Z}
$$

for every weight $w \in \mathfrak{t}^{*}$ of the representation of every field in the theory. This is simply the condition that the holonomy of $A$ around the point $\theta=\pi$ is trivial in the bundles of which the fields are sections. The theory always contains fields in the adjoint representation, so m belongs to the coweight lattice: ${ }^{2}$

$$
\mathbf{m} \in \Lambda_{\mathrm{cW}}(\mathfrak{g}) / W(G)
$$

Equivalently, $\mathbf{m}$ is specified by an irreducible representation of the Langlands dual ${ }^{L} \mathfrak{g}$ of g. In general, $\mathbf{m}$ lies in a sublattice of $\Lambda_{\mathrm{cw}}(\mathfrak{g}) / W(G)$ determined by the matter content.

\footnotetext{
${ }^{2}$ Further, $\mathbf{m}$ belongs to the cocharacter lattice $\left\{v \in \mathfrak{t} \mid \exp (2 \pi \mathrm{i} v)=\operatorname{id}_{G}\right\}$, which is a sublattice of $\Lambda_{\mathrm{cw}}(\mathfrak{g})$. If we take $G$ to be the adjoint group, the cocharacter lattice coincides with $\Lambda_{\mathrm{cw}}(\mathfrak{g})$.
} 
We can also consider heavy particles that carry both magnetic and electric charges. The worldline of such a dyon is called a Wilson-'t Hooft line. In the path integral formalism, a Wilson-'t Hooft line is realized by an insertion of a Wilson line

$$
\operatorname{Tr}_{R} P \exp \left(\mathrm{i} \int_{L} A\right)
$$

and a singular boundary condition on the support $L$ of the line as specified by the magnetic charge. The prescribed singularity (3.1) breaks the gauge symmetry to the stabilizer $G_{\mathbf{m}}$ of $\mathbf{m}$, so $R$ is an irreducible representation of $G_{\mathbf{m}}$. (More precisely, $R$ is an irreducible representation of the stabilizer of $\mathbf{m}$ in the universal cover $\widetilde{G}$ of $G$ [4].)

The data specifying such a pair $(\mathbf{m}, R)$ is actually the same as a pair $(\mathbf{m}, \mathbf{e})$ of coweight $\mathbf{m}$ and weight e modulo the Weyl group action:

$$
(\mathbf{m}, \mathbf{e}) \in\left(\Lambda_{\mathrm{cW}}(\mathfrak{g}) \times \Lambda_{\mathrm{w}}(\mathfrak{g})\right) / W(G)
$$

As emphasized in [4], this data has more information than a pair of irreducible representations of $\mathfrak{g}$ and ${ }^{L} \mathfrak{g}$.

In [10], the vevs of Wilson-'t Hooft lines in $\mathcal{N}=2$ supersymmetric gauge theories on $S^{1} \times \mathbb{R}^{2} \times \mathbb{R}$ in the Coulomb phase were computed via localization of the path integral. The geometry $S^{1} \times \mathbb{R}^{2}$ is a twisted product of $S^{1}$ and $\mathbb{R}^{2}$, constructed from $[0,2 \pi \beta] \times \mathbb{R}^{2}$ by the identification $(2 \pi \beta, z) \sim\left(0, e^{2 \pi \mathrm{i} \epsilon} z\right)$, where $z$ is the complex coordinate of $\mathbb{R}^{2} \cong \mathbb{C}$. These Wilson-'t Hooft lines wind around $S^{1}$, and are located at the origin of $\mathbb{R}^{2}$ and a point in $\mathbb{R}$. In order to preserve half of the eight supercharges, they require the complex scalar field $\phi$ in the vector multiplet to also have a singular behavior and replace the gauge field in the Wilson line (3.4) with $A+\mathrm{i} \operatorname{Re} \phi$. The vevs depend holomorphically on parameters

$$
a \in \mathfrak{t}_{\mathbb{C}}, \quad b \in \mathfrak{t}_{\mathbb{C}}^{*},
$$

which are set by the values of the gauge field and the vector multiplet scalar at spatial infinity. Essentially, $a$ is given by the holonomy around $S^{1}$ at infinity of the gauge field, while $b$ is that of the dual gauge field. ${ }^{3}$

The vev of a Wilson line $W_{R}$ in representation $R$ is simply given by the classical value of the holonomy:

$$
\left\langle W_{R}\right\rangle=\operatorname{Tr}_{R} e^{2 \pi \mathrm{i} a} .
$$

\footnotetext{
${ }^{3}$ Let $\theta_{\mathrm{e}}$ and $\theta_{\mathrm{m}}$ be the electric and magnetic theta-angles whose exponentials $e^{\mathrm{i} \theta_{\mathrm{e}}}$ and $e^{\mathrm{i} \theta_{\mathrm{m}}}$ are the electric and magnetic holonomies. (The magnetic holonomy can be defined as the chemical potential for the magnetic charge in the path integral.) Then, the parameters $a, b$ have semiclassical expansion

$$
a=\frac{\theta_{\mathrm{e}}}{2 \pi}+\mathrm{i} \beta \operatorname{Re} \phi+\cdots, \quad b=\frac{\theta_{\mathrm{m}}}{2 \pi}-\frac{4 \pi \mathrm{i} \beta}{g^{2}} \operatorname{Im} \phi+\mathrm{i} \frac{\vartheta}{2 \pi} \beta \operatorname{Re} \phi+\cdots,
$$

where $g$ is the gauge coupling and $\vartheta$ is the gauge theory theta-angle. These parameters are complexified Fenchel-Nielsen coordinates on the Seiberg-Witten moduli space and receive nonperturbative corrections (indicated above by the ellipses) which are known [29]. See [30] for a recent discussion on the nonperturbative corrections in the present context.
} 
The vevs of 't Hooft lines are much more involved. For an 't Hooft line $T_{\mathbf{m}}$ with magnetic charge $\mathbf{m}$, the vev takes the form

$$
\left\langle T_{\mathbf{m}}\right\rangle=\sum_{\substack{v \in \Lambda_{\text {cr }}(\mathfrak{g})+\mathbf{m} \\\|v\| \leq\|\mathbf{m}\|}} e^{2 \pi \mathrm{i}\langle v, b\rangle} Z_{1-\mathrm{loop}}(a, m, \epsilon ; v) Z_{\text {mono }}(a, m, \epsilon ; \mathbf{m}, v),
$$

where $m$ collectively denotes complex mass parameters. The summation over the coweights $v$ in the shifted coroot lattice $\Lambda_{\text {cr }}+\mathbf{m}$ accounts for the so-called "monopole bubbling," a phenomenon in which smooth monopoles are absorbed by the 't Hooft line and screen the magnetic charge. The norm $\|v\|$ with respect to a Killing form is bounded by $\|\mathbf{m}\|$, so this is a finite sum. The first two factors in the summand are the classical action and the one-loop determinant in the screened monopole background, respectively. The last factor is the nonperturbative contributions coming from degrees of freedom trapped on the 't Hooft line due to monopole bubbling.

Suppose that the theory under consideration consists of a vector multiplet and $N_{F}$ hypermultiplets in representations $R_{f}$ with mass parameters $m_{f}, f=1, \ldots, N_{F}$. The oneloop determinant $Z_{1 \text {-loop }}$ is then the product of the contributions from the vector multiplet and the hypermultiplets:

$$
Z_{1 \text {-loop }}(a, m, \epsilon ; v)=Z_{1-\text { loop }}^{\mathrm{vm}}(a, \epsilon ; v) \prod_{f=1}^{N_{F}} Z_{1 \text { loop }}^{\mathrm{hm}, R_{f}}\left(a, m_{f}, \epsilon ; v\right) \text {. }
$$

The two functions are given by

$$
\begin{aligned}
Z_{1-\text { loop }}^{\mathrm{vm}}(a, \epsilon ; v) & =\prod_{\alpha \in \Phi(\mathfrak{g})} \prod_{k=0}^{|\langle v, \alpha\rangle|-1} \sin ^{-\frac{1}{2}}\left(\pi\langle a, \alpha\rangle+\pi\left(\frac{1}{2}|\langle v, \alpha\rangle|-k\right) \epsilon\right) \\
Z_{1-\text { loop }}^{\mathrm{hm}, R}(a, m, \epsilon ; v) & =\prod_{w \in P(R)} \prod_{k=0}^{|\langle v, w\rangle|-1} \sin ^{\frac{1}{2}}\left(\pi\langle a, w\rangle-\pi m+\pi\left(\frac{1}{2}|\langle v, w\rangle|-\frac{1}{2}-k\right) \epsilon\right) .
\end{aligned}
$$

Here, $\Phi(\mathfrak{g})$ is the set of roots of $\mathfrak{g}$ and $P(R)$ is the set of weights of $R$.

The factor $Z_{\text {mono }}$ is subtle. The original computation in [10] did not give an answer that completely matches predictions from the AGT correspondence. The subtleties have been addressed in subsequent works [31-34] but not resolved in full generality.

Fortunately, for Wilson-'t Hooft lines that are of interest to us, the screened magnetic charges are in the same $W(G)$-orbit as $\mathbf{m}$. The corresponding contributions are therefore obtained by the $W(G)$-action from the perturbative term, for which $v=\mathbf{m}$ and $Z_{\text {mono }}=1$.

To our knowledge, a formula for the vevs of dyonic Wilson-'t Hooft lines generalizing the expressions (3.8) and (3.9) has not been derived. Nevertheless, for the same reason as mentioned, we can calculate the vev of a relevant Wilson-'t Hooft line by first writing down its perturbative contribution, which is simply the product of the perturbative vevs of the corresponding purely electric and purely magnetic lines, and then summing over the contributions from the nonperturbative sectors related by the $W(G)$-action. 


\subsection{Transfer matrices from circular quiver theories}

The Wilson-'t Hooft line that corresponds to the transfer matrix (2.43) is one in an $\mathcal{N}=2$ supersymmetric gauge theory that is described by a circular quiver with $n$ nodes:

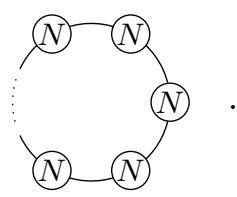

Each node represents a vector multiplet for an $\mathrm{SU}(N)$ gauge group,${ }^{4}$ and each edge a hypermultiplet that transforms in the bifundamental representation under the gauge groups of the nodes it connects.

Let us first consider the case in which the quiver consists of a single node and a single edge. In this case, the gauge group $G=\mathrm{SU}(N)$ and the only hypermultiplet is in the adjoint representation. This theory is known as $\mathcal{N}=2^{*}$ theory.

The roots of $\mathfrak{g}=\mathfrak{s u}_{N}$ are $\alpha_{i j}=E_{i i}^{*}-E_{j j}^{*}=h_{i}-h_{j}, i \neq j$. The positive roots are $\alpha_{i j}$, $i<j$, and the simple roots are $\alpha_{i}=\alpha_{i, i+1}, i=1, \ldots, N-1$. The fundamental coweights are $\omega_{i}^{\vee}=\left(\alpha_{i}^{\vee}\right)^{*}=\sum_{j=1}^{i} h_{j}^{\vee}$, with

$$
h_{i}^{\vee}=E_{i i}-\frac{1}{N} \sum_{j=1}^{N} E_{j j} .
$$

The various lattices are

$$
\Lambda_{\mathrm{r}}=\bigoplus_{i=1}^{N-1} \mathbb{Z} \alpha_{i}, \quad \Lambda_{\mathrm{cr}}=\bigoplus_{i=1}^{N-1} \mathbb{Z} \alpha_{i}^{\vee}, \quad \Lambda_{\mathrm{w}}=\bigoplus_{i=1}^{N-1} \mathbb{Z} \omega_{i}, \quad \Lambda_{\mathrm{cw}}=\bigoplus_{i=1}^{N-1} \mathbb{Z} \omega_{i}^{\vee} .
$$

We recall that $\alpha_{i}^{\vee}$ are the simple coroots and $\omega_{i}=\left(\alpha_{i}^{\vee}\right)^{*}$ are the fundamental weights.

For $\mathcal{N}=2^{*}$ theory with $G=\mathrm{SU}(N)$, minimal magnetic charges are $\mathbf{m}=\omega_{1}^{\vee}=h_{1}^{\vee}$ and $\mathbf{m}=\omega_{N-1}^{\vee}=-h_{N}^{\vee}$. These magnetic charges are the highest weights of the fundamental representation and the antifundamental representation of ${ }^{L_{\mathfrak{s u}}}{ }_{N} \cong \mathfrak{s u}_{N}$, respectively.

Let us consider the 't Hooft line with $\mathbf{m}=h_{1}^{\vee}$. The vev of this 't Hooft line is expressed as a sum over the screened magnetic charges $v=h_{1}^{\vee}, h_{2}^{\vee}, \ldots, h_{N}^{\vee}$. The term for $v=h_{1}^{\vee}$ is the perturbative contribution and given by

$$
e^{2 \pi \mathrm{i} b_{1}} \prod_{j=2}^{N} \sin ^{-\frac{1}{2}}\left(\pi a_{1 j}+\frac{1}{2} \pi \epsilon\right) \sin ^{-\frac{1}{2}}\left(\pi a_{j 1}+\frac{1}{2} \pi \epsilon\right) \sin ^{\frac{1}{2}}\left(\pi a_{1 j}-\pi m\right) \sin ^{\frac{1}{2}}\left(\pi a_{j 1}-\pi m\right),
$$

where $a_{i}=\left\langle a, h_{i}\right\rangle, b_{i}=\left\langle h_{i}^{\vee}, b\right\rangle, a_{i j}=a_{i}-a_{j}$ and $m$ is the mass of the adjoint hypermultiplet. The other terms are related to this perturbative term by the Weyl group action which permutes $\left(h_{1}^{\vee}, \ldots, h_{N}^{\vee}\right)$, so we find

$$
\left\langle T_{h_{1}^{\vee}}\right\rangle=\sum_{i=1}^{N} e^{2 \pi \mathrm{i} b_{i}} \prod_{j(\neq i)}\left(\frac{\sin \pi\left(a_{i j}-m\right) \sin \pi\left(a_{j i}-m\right)}{\sin \pi\left(a_{i j}-\frac{1}{2} \epsilon\right) \sin \pi\left(a_{j i}-\frac{1}{2} \epsilon\right)}\right)^{\frac{1}{2}} .
$$

The vev of $T_{-h_{N}^{\vee}}$ is obtained from $\left\langle T_{h_{1}^{\vee}}\right\rangle$ by the replacement $b_{i} \rightarrow-b_{i}$.

\footnotetext{
${ }^{4}$ More precisely, the gauge group is a product of $\operatorname{PSU}(N)$.
} 
Now, let us turn to a circular quiver with $n$ nodes. For this theory, we have $G=$ $\mathrm{SU}(N)^{n}$ and $\Lambda_{\mathrm{cw}}(\mathfrak{g})=\Lambda_{\mathrm{cw}}\left(\mathfrak{s u}_{N}\right)^{\oplus n}$. We consider the 't Hooft line with

$$
\mathbf{m}=h_{1}^{\vee} \oplus \cdots \oplus h_{1}^{\vee}
$$

charged equally under the $\mathrm{SU}(N)$ factors of $G$. This time, the summation is over all coweights of the form $v=h_{i^{1}}^{\vee} \cdots \oplus h_{i^{n}}^{\vee}$. The perturbative term, for which $i^{1}=\cdots=i^{n}=1$, is given by

$$
\begin{aligned}
\prod_{r=1}^{n} e^{2 \pi \mathrm{i} b_{1}^{r}} \prod_{j=2}^{N} \sin ^{-\frac{1}{2}}\left(\pi a_{1 j}^{r}\right. & \left.+\frac{1}{2} \pi \epsilon\right) \sin ^{-\frac{1}{2}}\left(\pi a_{j 1}^{r}+\frac{1}{2} \pi \epsilon\right) \\
& \times \sin ^{\frac{1}{2}}\left(\pi\left(a_{j}^{r}-a_{1}^{r+1}\right)-\pi m^{r}\right) \sin ^{\frac{1}{2}}\left(\pi\left(a_{1}^{r}-a_{j}^{r+1}\right)-\pi m^{r}\right) .
\end{aligned}
$$

The superscript $r$ refers to the $r$ th $\mathrm{SU}(N)$ factor of $G$, with $a^{n+1}=a^{1}$. Collecting the contributions from the other coweights, we get

$$
\left\langle T_{h_{1}^{\vee} \oplus \cdots \oplus h_{1}^{\vee}}\right\rangle=\sum_{i^{1}, \ldots, i^{n}} \prod_{r=1}^{n} e^{2 \pi \mathrm{i} i_{i^{r}}^{r}} \ell_{m^{r}}\left(a^{r}, a^{r+1}\right)_{i^{r}}^{i^{r+1}},
$$

where we have used the functions (2.40).

Comparing this expression with the Wigner transform (2.43) of the trigonometric transfer matrix $\mathcal{T}_{\sigma, m}$, we see

$$
\left\langle T_{h_{1}^{\vee} \oplus \cdots \oplus h_{1}^{\vee}}\right\rangle=\left\langle\mathcal{T}_{(+, \ldots,+), m}\right\rangle=\left\langle\mathcal{T}_{(-, \ldots,-), m}\right\rangle
$$

under the obvious identification of parameters.

In order to reproduce $\left\langle\mathcal{T}_{\sigma, m}\right\rangle$ for a general choice of the signs $\sigma$, we add to the 't Hooft line the electric charge

$$
\mathbf{e}=\sum_{r=1}^{n} \sigma^{r} \frac{1}{2}\left(h_{1}^{r+1}-h_{1}^{r}\right)=\sum_{r=1}^{n}\left(\sigma^{r} 1-\sigma^{r+1} 1\right) \frac{1}{2} h_{1}^{r+1} .
$$

This electric charge is in a sense a minimal one that is compatible with the Dirac-SchwingerZwanziger quantization condition for locality: the charges $(\mathbf{m}, \mathbf{e})$ and $\left(\mathbf{m}^{\prime}, \mathbf{e}^{\prime}\right)$ of two dyons must satisfy $\left\langle\mathbf{m}, \mathbf{e}^{\prime}\right\rangle-\left\langle\mathbf{m}^{\prime}, \mathbf{e}\right\rangle \in \mathbb{Z}$. In section 4 , we will see the geometric meaning of this "minimality" in connection with the AGT correspondence.

The magnetic charge (3.18) breaks the gauge group to $\mathrm{S}(\mathrm{U}(1) \times \mathrm{U}(N-1))^{n}$, and we are turning on a Wilson line that is charged under the $\mathrm{U}(1)$ factors with charges proportional to $\left(\sigma^{r} 1-\sigma^{r+1} 1\right) / 2$. The Wilson line multiplies the perturbative term (3.19) by the phase factor

$$
\prod_{r=1}^{n} e^{\sigma^{r} \pi \mathrm{i}\left\langle a, h_{1}^{r+1}-h_{1}^{r}\right\rangle}=\prod_{r=1}^{n} e^{\sigma^{r} \pi \mathrm{i}\left(a_{1}^{r+1}-a_{1}^{r}\right)} .
$$

Hence, the term with $v=h_{i^{1}}^{\vee} \oplus \cdots \oplus h_{i^{n}}^{\vee}$ gets the phase factor $e^{\sigma^{r} \pi \mathrm{i}\left(a_{i^{r+1}}^{r+1}-a_{i^{r}}^{r}\right)}$, and the vev of this Wilson-'t Hooft line matches the Wigner transform of $\mathcal{T}_{\sigma, m}$. 


\subsection{Monodromy matrices from linear quiver theories}

We have considered the Wilson-'t Hooft lines in the circular quiver theory and showed that their vevs match the Wigner transforms of the trigonometric transfer matrices. What correspond to the monodromy matrices then? In view of the fact that summing over the weights of the representation $V=\mathbb{C}^{N}$ in the integrable model amounts to summing over the different screened magnetic charges, natural candidates are Wilson-'t Hooft lines in a theory described by a linear quiver with $n+1$ nodes:

$$
N-(N)-(N-\cdots-N-N \text {. }
$$

The leftmost and the rightmost nodes represent $\mathrm{SU}(N)$ flavor groups, which are not gauged.

In particular, we expect that the fundamental trigonometric L-operators (2.38) arise from the vevs of Wilson-'t Hooft lines of the theory of a bifundamental hypermultiplet:

$$
N \quad N \quad \text {. }
$$

Let us see if this is the case.

We introduce nondynamical vector multiplets for the $\mathrm{SU}(N)$ flavor groups, and consider the Wilson-'t Hooft lines with magnetic charge

$$
\mathbf{m}=h_{i}^{\vee} \oplus h_{j}^{\vee}
$$

and electric charges

$$
\mathbf{e}=\mp \frac{1}{2} h_{i} \oplus \pm \frac{1}{2} h_{j} .
$$

Note that the electric charges are fractional. The vevs of these Wilson-'t Hooft lines are

$$
e^{2 \pi \mathrm{i}\left(b_{i}^{1}+b_{j}^{2}\right)} e^{ \pm \pi \mathrm{i}\left(a_{j}^{2}-a_{i}^{1}\right)} \prod_{k(\neq i)} \prod_{l(\neq j)}\left(\sin \pi\left(a_{k}^{1}-a_{j}^{2}-m\right) \sin \pi\left(a_{i}^{1}-a_{l}^{2}-m\right)\right)^{\frac{1}{2}} .
$$

The vevs do not quite match the Wigner transforms $(2.39)$ of $\left(\mathcal{L}_{ \pm, m}\right)_{i}^{j}$. They differ by the factor in the denominator of the function (2.40).

This factor is the one-loop determinant associated with the first node; it would have been present had the $\mathrm{SU}(N)$ flavor group been gauged and the vector multiplet been dynamical. From the gauge theory point of view, it is natural to think of this factor as a weight accompanying the summation over the screened magnetic charges. On the integrable system side, we could as well omit the denominator in question from the definitions of the L-operators and adopt the convention that the same weight is included when operators are multiplied within $V$. The L-operators would still satisfy the RLL relation.

To get the monodromy matrix (2.42), we take $n$ L-operators and multiply them inside $V$. The gauge theory counterpart of this operation is to connect $n$ copies of the two-node quiver (3.25), in the presence of appropriate Wilson-'t Hooft lines of the type considered above, by identifying and gauging flavor nodes. This produces the $n+1$ node linear quiver (3.24) and the Wilson-'t Hooft lines with magnetic charge

$$
\mathbf{m}=h_{i^{1}}^{\vee} \oplus h_{1}^{\vee} \oplus h_{1}^{\vee} \oplus \cdots \oplus h_{1}^{\vee} \oplus h_{i^{n+1}}^{\vee}
$$


and electric charge

$$
\mathbf{e}=\sigma^{1} \frac{1}{2}\left(h_{1}^{2}-h_{i^{1}}^{1}\right)+\sum_{r=2}^{n-1} \sigma^{r} \frac{1}{2}\left(h_{1}^{r+1}-h_{1}^{r}\right)+\sigma^{n} \frac{1}{2}\left(h_{i^{n+1}}^{n+1}-h_{1}^{n}\right) .
$$

The vev of this Wilson-'t Hooft line reproduces the Wigner transform (2.42), except that a factor corresponding to the one-loop determinant for the vector multiplet for the first node is missing.

\subsection{Other representations}

The magnetic charge (3.18) of the above Wilson-'t Hooft lines is the highest weight of the representation $\left(\mathbb{C}^{N}\right)^{\oplus n}$ of the Langlands dual ${ }^{L} \mathfrak{g}_{\mathbb{C}} \cong \mathfrak{s l}_{N}^{\oplus n}$ of $\mathfrak{g}_{\mathbb{C}}$. The corresponding transfer matrix (2.43) is represented graphically as $n$ double lines intersected by a single solid loop carrying the representation $V=\mathbb{C}^{N}$, as shown in figure $1(\mathrm{c})$. The $n$ regions sandwiched between double lines correspond to the $n$ copies of $\mathfrak{s l}_{N}$.

Both sides of the correspondence have a generalization in which the vector representation $\mathbb{C}^{N}$ is replaced by another representation $R$ of $\mathfrak{s l}_{N}$. On the gauge theory side, we can change the magnetic charge of the Wilson-'t Hooft lines to the highest weight $\lambda_{R}$ of $R^{\oplus n}$ while keeping the electric charges intact. On the integrable system side, the counterpart of this operation is the fusion procedure, which allows one to construct a solid line in an arbitrary finite-dimensional representation of $\mathfrak{s l}_{N}$ from a collection of solid lines in the vector representation, with the spectral parameters suitably adjusted.

We naturally expect that the vev of the Wilson-'t Hooft line with magnetic charge $\mathbf{m}=\lambda_{R}^{\oplus n}$ is equal to the Wigner transform of a transfer matrix constructed from Loperators in representation $R$, obtained by fusion from the L-operators (2.38) in the vector representation.

For $n=1$ and $R=\wedge^{k} \mathbb{C}^{N}$, this equality can be verified from known results. In this case, the transfer matrix is the trigonometric limit of Ruijsenaars' difference operator [35]

$$
\sum_{\substack{I \subset\{1, \ldots, N\} \\|I|=k}} \Delta_{I}^{\frac{1}{2}} \prod_{\substack{i \in I \\ j \notin I}} \sqrt{\frac{\theta_{1}\left(a_{j i}-m\right) \theta_{1}\left(a_{i j}-m\right)}{\theta_{1}\left(a_{j i}-\frac{1}{2} \epsilon\right) \theta_{1}\left(a_{i j}-\frac{1}{2} \epsilon\right)}} \Delta_{I}^{\frac{1}{2}}, \quad \Delta_{I}=\prod_{i \in I} \Delta_{i},
$$

and is related to the Macdonald operator by a similarity transformation [5]. On the other hand, the exterior power $\wedge^{k} \mathbb{C}^{N}$ being a minuscule representation (that is, all weights are related by the action of the Weyl group), the vev of the 't Hooft line with $\mathbf{m}=\omega_{k}^{\vee}=$ $h_{1}^{\vee}+\cdots+h_{k}^{\vee}$ in $\mathcal{N}=2^{*}$ theory can be computed from the perturbative term:

$$
\left\langle T_{\omega_{k}^{\vee}}\right\rangle=\sum_{\substack{I \subset\{1, \ldots, N\} \\|I|=k}} \prod_{\substack{i \in I \\ j \notin I}} e^{2 \pi \mathrm{i} b_{i}}\left(\frac{\sin \pi\left(a_{i j}-m\right) \sin \pi\left(a_{j i}-m\right)}{\sin \pi\left(a_{i j}-\frac{1}{2} \epsilon\right) \sin \pi\left(a_{j i}-\frac{1}{2} \epsilon\right)}\right)^{\frac{1}{2}} .
$$

(For $\mathcal{N}=2^{*}$ theory the choice of the signs $\sigma= \pm$ is irrelevant.) The vev matches the Wigner transform of the trigonometric Ruijsenaars operator. 


\section{Transfer matrices from Verlinde operators}

We have computed the vevs of a class of Wilson-'t Hooft lines in $\mathcal{N}=2$ supersymmetric gauge theories described by a circular quiver, and found that they match the Wigner transforms of transfer matrices constructed from the fundamental trigonometric L-operators. In this section, we show that these transfer matrices can also be identified with Verlinde operators in Toda theory on a punctured torus. The result is in keeping with the relation proposed in [10] based on the AGT correspondence [11] between Toda theory and $\mathcal{N}=2$ supersymmetric field theories.

\subsection{Verlinde operators and Wilson-'t Hooft lines}

The AGT correspondence originates from six-dimensional $\mathcal{N}=(2,0)$ supersymmetric field theory, of type $A_{N-1}$ in our case, placed on $S_{\mathrm{b}}^{4} \times C_{g, n}$. Here, $S_{\mathrm{b}}^{4}$ is an ellipsoid, defined as a submanifold of $\mathbb{R}^{5}$ by the equation

$$
\left(x^{1}\right)^{2}+\mathrm{b}^{-2}\left(\left(x^{2}\right)^{2}+\left(x^{3}\right)^{2}\right)+\mathrm{b}^{2}\left(\left(x^{4}\right)^{2}+\left(x^{5}\right)^{2}\right)=r^{2},
$$

and $C_{g, n}$ is a Riemann surface of genus $g$ with $n$ punctures. With partial topological twisting along $C_{g, n}$, this system preserves eight of the sixteen supercharges of $\mathcal{N}=(2,0)$ supersymmetry in six dimensions.

In the limit in which $C_{g, n}$ shrinks to a point, the six-dimensional theory reduces to a four-dimensional $\mathcal{N}=2$ supersymmetric field theory on $S_{\mathrm{b}}^{4}$, whose gauge and matter contents are determined by the choice of a pants decomposition of $C_{g, n}$ and boundary conditions at the punctures [36, 37]. The theories discussed in section 3 can all be obtained in this way. If one instead integrates out the modes along $S_{\mathrm{b}}^{4}$, one is left with $A_{N-1}$ Toda theory on $C_{g, n}$ with central charge

$$
c=1+6 q^{2}, \quad q=\mathrm{b}+\mathrm{b}^{-1},
$$

with vertex operators $V_{\beta^{r}}, r=1, \ldots, n$, inserted at the punctures. According to the AGT correspondence, the partition function of the theory on $S_{\mathrm{b}}^{4}$ equals the correlation function of Toda theory on $C_{g, n}$ :

$$
\langle 1\rangle_{S_{\mathrm{b}}^{4}}=\left\langle\prod_{r} V_{\beta^{r}}\right\rangle_{C_{g, n}}
$$

Let the vertex operators at the punctures be primary fields $V_{\beta^{r}}, r=1, \ldots, n$, labeled by momenta $\beta^{r} \in \mathfrak{h}^{*}$ valued in the dual of the Cartan subalgebra $\mathfrak{h}$ of $\mathfrak{s l}_{N}$. Given a pants decomposition of $C_{g, n}$, the Toda correlation function takes the form

$$
\left\langle\prod_{r} V_{\beta^{r}}\right\rangle_{C_{g, n}}=\int[\mathrm{d} \alpha] \mathcal{C}(\alpha ; \beta) \overline{\mathcal{F}(\alpha ; \beta)} \mathcal{F}(\alpha ; \beta),
$$

where $[\mathrm{d} \alpha]$ is a measure of integration over the set $\alpha=\left\{\alpha^{1}, \ldots, \alpha^{3 g-3+n}\right\}$ of momenta assigned to the internal edges of the pants decomposition, $\beta=\left\{\beta^{1}, \ldots, \beta^{n}\right\}$ is the set of momenta assigned to the external edges, $\mathcal{C}(\alpha ; \beta)$ is the product of relevant three-point functions, and $\mathcal{F}(\alpha ; \beta)$ is the corresponding conformal block which is a meromorphic function of $\alpha$ and $\beta$. 
On the gauge theory side, $\mathcal{C}(\alpha ; \beta)$ is interpreted as the product of the classical and the one-loop contributions to the partition function on $S_{\mathrm{b}}^{4}$, whereas $\mathcal{F}(\alpha ; \beta)$ and $\overline{\mathcal{F}(\alpha ; \beta)}$ represent the nonperturbative contributions from instantons localized at the two poles at $x^{2}=x^{3}=x^{4}=x^{5}=0$. The internal momenta $\alpha$ are related to the zero modes a of scalar fields in the vector multiplets by

$$
\alpha=Q+\text { ia , }
$$

and the external momenta $\beta$ are identified with mass parameters for matter multiplets.

To incorporate Wilson-'t Hooft lines in the gauge theory, one introduces Verlinde loop operators in the Toda theory. We will explain the construction of relevant Verlinde operators in concrete examples. For the moment, it suffices to say that they are specified by a momentum of the form $\mu=-\mathrm{b} \lambda$ and a one-cycle $\gamma$ in $C_{g, n}$, where $\lambda$ is the highest weight of a representation of $\mathfrak{s l}_{N}{ }^{5}$ In the presence of a Verlinde operator $\Phi_{\mu}(\gamma)$, the Toda correlation function is modified to

$$
\left\langle\Phi_{\mu}(\gamma) \prod_{r} V_{\beta^{r}}\right\rangle_{C_{g, n}}=\int[\mathrm{d} \alpha] \mathcal{C}(\alpha ; \beta) \overline{\mathcal{F}(\alpha ; \beta)}\left(\Phi_{\mu}(\gamma) \cdot \mathcal{F}(\alpha ; \beta)\right) .
$$

The AGT correspondence asserts $[13,14]$ that this is equal to the vev of a Wilson-' $t$ Hooft line $T_{\mu, \gamma}$ winding around a circle $S_{\mathrm{b}}^{1}$ where $x^{4}=x^{5}=0$ (at $x^{1}=0$, say):

$$
\left\langle T_{\mu, \gamma}\right\rangle_{S_{\mathrm{b}}^{4}}=\left\langle\Phi_{\mu}(\gamma) \prod_{r} V_{\beta^{r}}\right\rangle_{C_{g, n}}
$$

It turns out that $\Phi_{\mu}(\gamma)$ acts on conformal blocks as a difference operator shifting the internal momenta $\alpha$, just as Wilson-'t Hooft lines in $\mathcal{N}=2$ supersymmetric gauge theories on $S^{1} \times_{\epsilon} \mathbb{R}^{2} \times \mathbb{R}$ shift Coulomb branch parameters. Indeed, it was argued in [10] that if one defines the modified Verlinde operator

$$
\mathcal{L}_{\mu}(\gamma)=\mathcal{C}(\alpha ; \beta)^{\frac{1}{2}} \Phi_{\mu}(\gamma) \mathcal{C}(\alpha ; \beta)^{-\frac{1}{2}}
$$

then its Wigner transform is equal to the vev of the Wilson-'t Hooft line in the theory on $S^{1} \times \mathbb{R}^{2} \times \mathbb{R}$, up to an appropriate identification of parameters:

$$
\left\langle T_{\mu, \gamma}\right\rangle_{S^{1} \times \mathbb{R}^{2} \times \mathbb{R}}=\left\langle\mathcal{L}_{\mu}(\gamma)\right\rangle .
$$

Therefore, we expect that for suitable choices of $C_{g, n}, \beta, \mu$ and $\gamma$, the modified Verlinde operator $\mathcal{L}_{\mu}(\gamma)$ coincides with a transfer matrix constructed from the trigonometric Loperator.

\subsection{Verlinde operators on a punctured torus}

To reproduce the transfer matrix (2.43), we consider Toda theory on an $n$-punctured torus $C_{1, n}$ and insert vertex operators $V_{\beta^{r}}$ with

$$
\beta^{r}=-N\left(\frac{q}{2}+\mathrm{im}^{r}\right) h_{N} .
$$

\footnotetext{
${ }^{5}$ More generally, the momentum takes the form $\mu=-b \lambda_{1}-b^{-1} \lambda_{2}$, where $\lambda_{1}, \lambda_{2}$ are the highest weights of a pair of representations of $\mathfrak{s l}_{N}$. The corresponding Wilson-'t Hooft line is a superposition of lines wrapping $S_{\mathrm{b}}^{1}$ and another circle $S_{\mathrm{b}^{-1}}^{1}$ where $x^{2}=x^{3}=0$.
} 


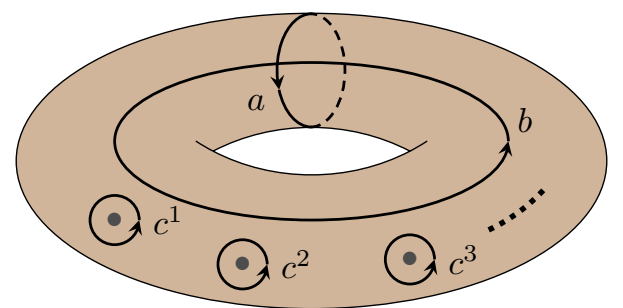

Figure 2. One-cycles on a punctured torus. The cycle $c^{r}$ goes around the $r$ th puncture.

The corresponding four-dimensional theory on $S_{\mathrm{b}}^{4}$ is the one described by an $n$-node circular quiver, which we studied in section 3.2. The parameter $\mathrm{m}^{r}$ is the mass of the bifundamental hypermultiplet between the $r$ th and $(r+1)$ th nodes.

To this setup we introduce the Verlinde operator $\Phi_{\mu}(\gamma)$ with

$$
\mu=-\mathrm{b} \omega_{1}=-\mathrm{b} h_{1}
$$

and $\gamma$ being a cycle $\gamma_{\sigma}$ specified by an $n$-tuple of signs $\sigma \in\{ \pm\}^{n}$. If $b$ and $c^{r}$ are the cycles shown in figure 2 , then

$$
\gamma_{\sigma}=b+\sum_{r} \frac{1-\sigma^{r} 1}{2} c^{r} .
$$

In other words, the curve $\gamma_{\sigma}$ passes "above" or "below" the $r$ th puncture depending on whether $\sigma^{r}=+$ or - . In the gauge theory, this operator corresponds to the Wilson-'t Hooft line with magnetic charge (3.18) and electric charge (3.22).

Let us explain the construction of this Verlinde operator step by step, following the treatment in [15]. To this end, it is convenient to represent the conformal block graphically as

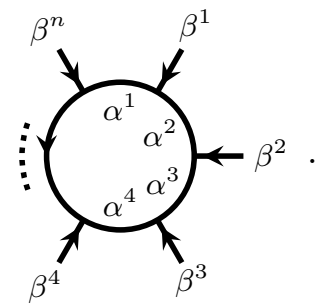

The internal momenta are $\alpha^{r}, r=1, \ldots, n+1$, with $\alpha^{n+1}=\alpha^{1}$.

The first step is to insert the identity operator between $\beta^{n}$ and $\beta^{1}$, and resolve it into the chiral vertex operators $V_{-\mathrm{b} h_{1}}$ and $V_{\mathrm{b} h_{N}}$ by fusion. This step gives the equality

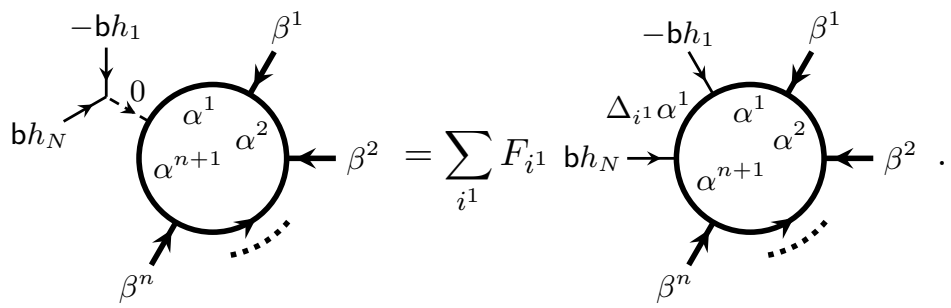

The difference operator $\Delta_{i}$ acts on internal momenta by

$$
\Delta_{i} \alpha=\alpha-\mathrm{b} h_{i} .
$$


The function $F_{i^{1}}$ is given by

$$
F_{i^{1}}=\frac{\Gamma(N \mathrm{~b} q)}{\Gamma(\mathrm{b} q)} \prod_{j^{1}\left(\neq i^{1}\right)} \frac{\Gamma\left(\mathrm{iba}_{j^{1} i^{1}}^{1}\right)}{\Gamma\left(\mathrm{b} q+\mathrm{iba}_{j^{1} i^{1}}^{1}\right)},
$$

with

$$
Q=q \rho, \quad \rho=\sum_{i=1}^{N-1} \omega_{i} .
$$

Next, we transport $V_{-\mathrm{b} h_{1}}$ along $\gamma_{\sigma}$. Graphically, we move the external edge labeled $-\mathrm{b} h_{1}$ clockwise. Every time the line passes another external edge we get a braiding factor:

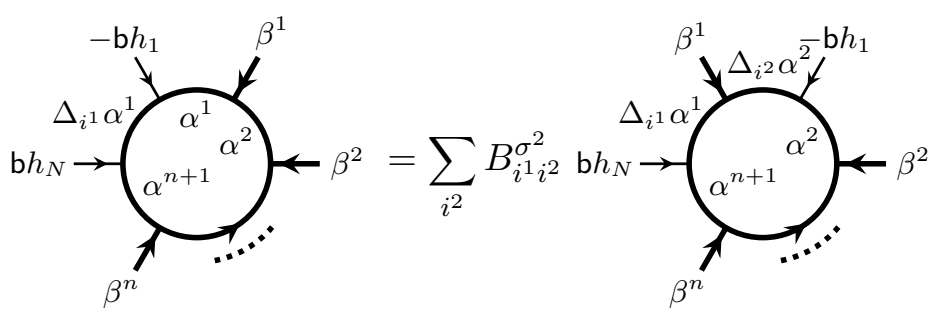

$$
=\sum_{i^{2}, \ldots, i^{n+1}}\left(\prod_{r=1}^{n} B_{i^{r} i^{r+1}}^{\sigma^{r}}\right) \underset{\Delta_{i^{n+1}} \alpha^{n+1}}{\beta_{\beta^{n}}}
$$

The function $B_{i^{r} i^{r+1}}^{\sigma^{r}}$ depends on the sign $\sigma^{r}$, which specifies the direction of the braiding moves:

$$
\begin{aligned}
B_{i^{r} i^{r+1}}^{\sigma^{r}}= & e^{-\sigma^{r} \pi \mathrm{b}\left(\mathrm{a}_{i^{r}}^{r}-\mathrm{a}_{i^{r+1}}^{r+1}\right)} \prod_{j^{r}\left(\neq i^{r}\right)} \frac{\Gamma\left(\mathrm{b}\left(q+\mathrm{ia}_{j^{r} i^{r}}^{r}\right)\right)}{\Gamma\left(\mathrm{b}\left(\frac{1}{2} q+\mathrm{ia}_{j^{r}}^{r}-\mathrm{ia}_{i^{r+1}}^{r+1}-\mathrm{im}^{r}\right)\right)} \\
& \times \prod_{j^{r+1}\left(\neq i^{r+1}\right)} \frac{\Gamma\left(\mathrm{iba}_{j^{r+1} i^{r+1}}^{r+1}\right)}{\Gamma\left(\mathrm{b}\left(\frac{1}{2} q+\mathrm{ia}_{j^{r+1}}^{r+1}-\mathrm{ia}_{i^{r}}^{r}+\mathrm{im}^{r}\right)\right)}
\end{aligned}
$$

Finally, we fuse $V_{-\mathrm{b} h_{1}}$ and $V_{\mathrm{b} h_{N}}$ and project the result to the channel in which the intermediate state is the identity operator:

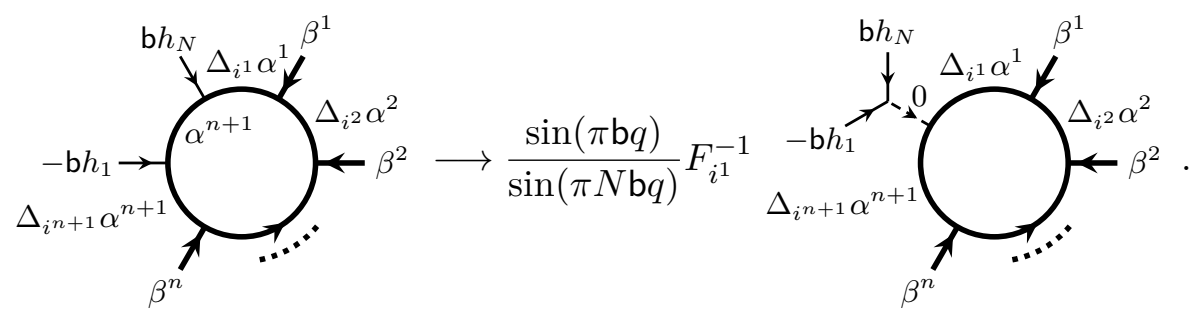

Note that the right-hand side vanishes unless $i^{n+1}=i^{1}$ since $\alpha^{n+1}=\alpha^{1}$. 
Thus, dropping the overall factor $\sin (\pi \mathrm{b} q) / \sin (\pi N \mathrm{~b} q)$, we find that the Verlinde operator is the difference operator

$$
\Phi_{-\mathrm{b} h_{1}}\left(\gamma_{\sigma}\right)=\sum_{i^{1}, \ldots, i^{n}}\left(\prod_{r} B_{i^{r} i^{r+1}}^{\sigma^{r}}\right) \Delta_{\left\{i^{1}, \ldots, i^{n}\right\}}
$$

where $i^{n+1}=i^{1}$ and

$$
\Delta_{\left\{i^{1}, \ldots, i^{n}\right\}}=\prod_{r} \Delta_{i^{r}}^{r}
$$

Before we compare the Verlinde operator with the transfer matrix, we must perform a change of basis and find the modified Verlinde operator (4.8). For the correlation function at hand, the product of three-point function factors is

$$
\mathcal{C}(\alpha ; \beta)=\prod_{r} \frac{\prod_{i<j} \Upsilon\left(\mathrm{ia}_{i j}^{r}\right) \Upsilon\left(-\mathrm{ia}_{i j}^{r+1}\right)}{\prod_{i, j} \Upsilon\left(\frac{1}{2} q+\mathrm{im}^{r}-\mathrm{ia}_{i}^{r}+\mathrm{ia}_{j}^{r+1}\right)} .
$$

The precise definition of the function $\Upsilon$ is not important for us; we just need to know that it satisfies the identity

$$
\frac{\Upsilon(x+b)}{\Upsilon(x)}=\frac{\Gamma(b x)}{\Gamma(1-b x)} b^{1-2 b x},
$$

where $\Gamma$ is the gamma function.

Let us calculate $\mathcal{C}(\alpha ; \beta) \Delta_{\left\{i^{1}, \ldots, i^{n}\right\}} \mathcal{C}(\alpha ; \beta)^{-1}$. The only nontrivial contributions come from the $\Upsilon$-factors in which either of $i$ or $j$ (but not both) in $\mathrm{a}_{i j}^{r}$ is equal to $i^{r}$ :

$$
\begin{aligned}
\mathcal{C}(\alpha ; \beta) & \Delta_{\left\{i^{1}, \ldots, i^{n}\right\}} \mathcal{C}(\alpha ; \beta)^{-1} \\
= & \prod_{r} \prod_{\left(i^{r}<\right) j} \frac{\Upsilon\left(\mathrm{ia}_{i^{r} j}^{r}\right)}{\Upsilon\left(\mathrm{ia}_{i^{r} j}^{r}-\mathrm{b}\right)} \prod_{i\left(<i^{r}\right)} \frac{\Upsilon\left(\mathrm{ia}_{i i^{r}}^{r}\right)}{\Upsilon\left(\mathrm{ia}_{i i^{r}}^{r}+\mathrm{b}\right)} \\
& \times \prod_{\left(i^{r+1}<\right) j} \frac{\Upsilon\left(-\mathrm{ia} i^{r+1} j\right)}{\Upsilon\left(-\mathrm{ia}_{i^{r+1} j}^{r+1}+\mathrm{b}\right)} \prod_{i\left(<i^{r+1}\right)} \frac{\Upsilon\left(-\mathrm{ia}_{i i^{+1}}^{r+1}\right)}{\Upsilon\left(-\mathrm{ia}_{i i^{r+1}}^{r+1}-\mathrm{b}\right)} \\
& \times \prod_{i\left(\neq i^{r}\right)} \frac{\Upsilon\left(\frac{1}{2} q+\mathrm{im}^{r}-\mathrm{ia}_{i}^{r}+\mathrm{ia}_{i^{r+1}}^{r+1}-\mathrm{b}\right)}{\Upsilon\left(\frac{1}{2} q+\mathrm{im}^{r}-\mathrm{ia}_{i}^{r}+\mathrm{ia}_{i^{r+1}}^{r+1}\right)} \prod_{j\left(\neq i^{r+1}\right)} \frac{\Upsilon\left(\frac{1}{2} q+\mathrm{im}^{r}-\mathrm{ia}_{i^{r}}^{r}+\mathrm{ia}_{j}^{r+1}+\mathrm{b}\right)}{\Upsilon\left(\frac{1}{2} q+\mathrm{im}^{r}-\mathrm{ia}_{i^{r}}^{r}+\mathrm{ia}_{j}^{r+1}\right)} .
\end{aligned}
$$

Combining the first two lines and using the aforementioned identity, we can rewrite this quantity as

$$
\begin{aligned}
\mathcal{C}(\alpha ; \beta) & \Delta_{\left\{i^{1}, \ldots, i^{n}\right\}} \mathcal{C}(\alpha ; \beta)^{-1} \\
= & \prod_{r} \prod_{j^{r}\left(\neq i^{r}\right)} \frac{\Gamma\left(1-\mathrm{b}\left(q+\mathrm{ia}_{j^{r} i^{r}}^{r}\right)\right) \Gamma\left(\mathrm{b}\left(\frac{1}{2} q+\mathrm{ia}_{j^{r}}^{r}-\mathrm{ia}_{i^{r+1}}^{r+1}-\mathrm{im}^{r}\right)\right)}{\Gamma\left(\mathrm{b}\left(q+\mathrm{ia}_{j^{r} i^{r}}^{r}\right)\right) \Gamma\left(1-\mathrm{b}\left(\frac{1}{2} q+\mathrm{ia}_{j^{r}}^{r}-\mathrm{ia}_{i^{r+1}}^{r+1}-\mathrm{im}^{r}\right)\right)} \\
& \times \prod_{j^{r+1}\left(\neq i^{r+1}\right)} \frac{\Gamma\left(1-\mathrm{iba}_{j^{r+1} i^{r+1}}^{r+1}\right) \Gamma\left(\mathrm{b}\left(\frac{1}{2} q+\mathrm{ia}_{j^{r+1}}^{r+1}-\mathrm{ia}_{i^{r}}^{r}+\mathrm{im}^{r}\right)\right)}{\Gamma\left(\mathrm{iba}_{j^{r+1} i^{r+1}}^{r+1}\right) \Gamma\left(1-\mathrm{b}\left(\frac{1}{2} q+\mathrm{ia}_{j^{r+1}}^{r+1}-\mathrm{ia}_{i^{r}}^{r}+\mathrm{im}^{r}\right)\right)} .
\end{aligned}
$$

Plugging this expression into the formula for the modified Verlinde operator, we see that the various factors of gamma functions combine nicely into sine functions via Euler's reflection formula

$$
\Gamma(x) \Gamma(1-x)=\frac{\pi}{\sin (\pi x)} .
$$


The final result is

$$
\begin{aligned}
\mathcal{L}_{-\mathrm{b} h_{1}}\left(\gamma_{\sigma}\right)=\sum_{i^{1}, \ldots, i^{n}} & \left(\prod_{r} e^{-\sigma^{r} \pi \mathrm{b}\left(\mathrm{a}_{i^{r}}^{r}-\mathrm{a}_{i^{r+1}}^{r+1}\right)} \prod_{j^{r}\left(\neq i^{r}\right)}\left(\frac{\sin \pi \mathrm{b}\left(\frac{1}{2} q+\mathrm{ia}_{j^{r}}^{r}-\mathrm{ia}_{i^{r+1}}^{r+1}-\mathrm{im}^{r}\right)}{\sin \pi \mathrm{b}\left(q+\mathrm{ia}_{j^{r} i^{r}}^{r}\right)}\right)^{\frac{1}{2}}\right. \\
& \left.\times \prod_{j^{r+1}\left(\neq i^{r+1}\right)}\left(\frac{\sin \pi \mathrm{b}\left(\frac{1}{2} q+\mathrm{ia}_{j^{r+1}}^{r+1}-\mathrm{ia}_{i^{r}}^{r}+\mathrm{im}^{r}\right)}{\sin \pi \mathrm{iba}_{j^{r+1} i^{r+1}}^{r+1}}\right)^{\frac{1}{2}}\right) \Delta_{\left\{i^{1}, \ldots, i^{n}\right\}} .
\end{aligned}
$$

The above expression can be written in terms of the functions (2.40) as

$$
\begin{aligned}
\mathcal{L}_{-\mathrm{b} h_{1}}\left(\gamma_{\sigma}\right) & \\
& =\sum_{i^{1}, \ldots, i^{n}} \Delta_{\left\{i^{1}, \ldots, i^{n}\right\}}^{\frac{1}{2}}\left(\prod_{r} \ell_{\mathrm{ibm}^{r}+\frac{1}{2}}\left(\mathrm{iba}^{r}, \mathrm{iba}^{r+1}\right)_{i^{r}}^{i^{r+1}} e^{\sigma^{r} \pi \mathrm{i}\left(\mathrm{iba}_{i^{r}}^{r}-\mathrm{iba}_{i^{r+1}}^{r+1}\right)}\right) \Delta_{\left\{i^{1}, \ldots, i^{n}\right\}}^{\frac{1}{2}} .
\end{aligned}
$$

Comparing this expression with the Wigner transform (2.43) of the trigonometric transfer matrix, we deduce that the modified Verlinde operator coincides with the transfer matrix,

$$
\mathcal{L}_{-\mathrm{b} h_{1}}\left(\gamma_{\sigma}\right)=\mathcal{T}_{\sigma, m}
$$

under the identification

$$
\epsilon=\mathrm{b}^{2}, \quad a^{r}=\mathrm{iba}^{r}, \quad m^{r}=\mathrm{ibm}^{r}+\frac{1}{2} .
$$

It has been proposed in [10] that precisely under this identification of parameters, a modified Verlinde operator in Toda theory corresponding to a Wilson-'t Hooft line in the AGT-dual theory on $S_{\mathrm{b}}^{4}$ reproduces the Weyl quantization of the same Wilson-'t Hooft line in the same theory, but placed in the spacetime $S^{1} \times_{\epsilon} \mathbb{R}^{2} \times \mathbb{R}$. Therefore, we again reach the conclusion that the vev of the Wilson-'t Hooft line with charge (3.18) and (3.22) are equal to the Wigner transform of the trigonometric transfer matrix (2.43).

\section{Brane realization}

The AGT correspondence between Wilson-'t Hooft lines and Verlinde operators, which we exploited in section 4, can be realized in terms of branes in string theory. String dualities relate the brane configuration for the AGT correspondence to another configuration that realizes four-dimensional Chern-Simons theory, and in the latter setup the emergence of quantum integrability can be seen more transparently. Another chain of dualities relate these setups to the one studied in $[2,3]$, which provided the initial motivation for the present work. In this last section we discuss these brane constructions.

As explained in section 4, the field theoretic origin of the AGT correspondence is sixdimensional $\mathcal{N}=(2,0)$ superconformal field theory, which in our context is of type $A_{N-1}$ and compactified on an $n$-punctured torus $C_{1, n}$. This theory describes the low-energy dynamics of a stack of $N$ M5-branes (modulo the center-of-mass degrees of freedom), intersected by $n$ M5-branes. 
Consider M-theory in the eleven-dimensional spacetime

$$
M_{11}=\mathbb{R}_{0} \times \mathbb{R}_{12}^{2} \times_{\epsilon} S_{3}^{1} \times_{-\epsilon} \mathbb{R}_{45}^{2} \times S_{6}^{1} \times \mathbb{R}_{7} \times \mathbb{R}_{8} \times \mathbb{R}_{9} \times S_{10}^{1} .
$$

(The subscripts indicate the directions in which the spaces extend.) We put $N$ M5-branes $\mathrm{M} 5_{i}, i=1, \ldots, N$, on

$$
M_{\mathrm{M} 5_{i}}=\mathbb{R}_{0} \times \mathbb{R}_{12}^{2} \times{ }_{\epsilon} S_{3}^{1} \times\{0\} \times S_{6}^{1} \times\{0\} \times\{0\} \times\{0\} \times S_{10}^{1} .
$$

They realize $\mathcal{N}=(2,0)$ superconformal field theory on $\mathbb{R}_{0} \times \mathbb{R}_{12}^{2} \times_{\epsilon} S_{3}^{1} \times C_{1}$, with

$$
C_{1}=S_{6}^{1} \times S_{10}^{1}
$$

Further, we introduce $n$ M5-branes $\mathrm{M}^{r}, r=1, \ldots, n$, with worldvolumes

$$
M_{\mathrm{M}^{r}}=\mathbb{R}_{0} \times \mathbb{R}_{12}^{2} \times{ }_{\epsilon} S_{3}^{1} \times\{0\} \times\left\{l^{r}\right\} \times\{0\} \times \mathbb{R}_{8} \times \mathbb{R}_{9} \times\left\{\theta^{r}\right\} .
$$

These M5-branes create codimension-two defects in the six-dimensional theory, located at $n$ points $\left(l^{r}, \theta^{r}\right)$ on $C_{1}$, making $C_{1}$ an $n$-punctured torus $C_{1, n}$.

The two sets of M5-branes share a four-dimensional part of the spacetime, $\mathbb{R}_{0} \times \mathbb{R}_{12}^{2} \times \epsilon$ $S_{3}^{1}$, and on this four-dimensional spacetime we get an $\mathcal{N}=2$ supersymmetric gauge theory with gauge group $G=\mathrm{SU}(N)^{n}$, described by the circular quiver with $n$ nodes. (More precisely, the gauge group is $\mathrm{SU}(N)^{n} \times \mathrm{U}(1)$ but the $\mathrm{U}(1)$ factor is associated with the center-of-mass and decoupled from the rest of the theory.) In fact, reduction on $S_{10}^{1}$ turns $\mathrm{M} 5_{i}$ into D4-branes $\mathrm{D} 4_{i}$ on

$$
M_{\mathrm{D} 4_{i}}=\mathbb{R}_{0} \times \mathbb{R}_{12}^{2} \times_{\epsilon} S_{3}^{1} \times\{0\} \times S_{6}^{1} \times\{0\} \times\{0\} \times\{0\}
$$

and $\mathrm{M} 5^{r}$ into NS5-branes NS5 ${ }^{r}$ on

$$
M_{\mathrm{NS} 5}{ }^{r}=\mathbb{R}_{0} \times \mathbb{R}_{12}^{2} \times{ }_{\epsilon} S_{3}^{1} \times\{0\} \times\left\{l^{r}\right\} \times\{0\} \times \mathbb{R}_{8} \times \mathbb{R}_{9},
$$

and the above brane configuration becomes the well-known D4-NS5 brane configuration for the circular quiver theory [38]. The difference $l^{r+1}-l^{r}$ in the $x^{6}$-coordinate between $\mathrm{NS}^{r+1}$ and $\mathrm{NS}^{r}$ is inversely proportional to the square of the gauge coupling for the $r$ th gauge group, whereas the difference $\theta^{r+1}-\theta^{r}$ in the $x^{10}$-coordinate is the theta-angle for the $r$ th gauge group. ${ }^{6}$

A Wilson-'t Hooft line in this four-dimensional theory is realized by an M2-brane on

$$
M_{\mathrm{M} 2}=\left\{t_{0}\right\} \times\{0\} \times S_{3}^{1} \times\{0\} \times S_{6}^{1} \times\{0\} \times \mathbb{R}_{8}^{\geq 0} \times\left\{x_{0}\right\} \times\left\{\theta_{0}\right\},
$$

\footnotetext{
${ }^{6}$ To realize nonzero values for the parameters $a^{r}$ and $b^{r}$, we break each D4-brane $\mathrm{D} 4_{i}$ into $n$ segments $\mathrm{D} 4_{i}^{r}$ suspended between neighboring NS5-branes and allow these segments to be located anywhere on $\mathbb{R}_{8} \times \mathbb{R}_{9}$. Then, $a_{i}^{r}$ is a complex linear combination of the $x^{9}$-coordinate of $\mathrm{D} 4_{i}^{r}$ and the background holonomy of the U(1) gauge field on $\mathrm{D} 4_{i}^{r}$ around $S_{3}^{1}$. The definition of $b_{i}^{r}$ is similar, but involves both the $x^{8}$ - and $x^{9}$-coordinates as well as a chemical potential for the magnetic charge at infinity which does not have a simple interpretation in this brane system.
} 
where $\mathbb{R}_{\overline{8}}^{\geq 0}$ is the nonnegative part of $\mathbb{R}_{8}$. Upon reduction on $S_{10}^{1}$, this M2-brane becomes a D2-brane on

$$
M_{\mathrm{D} 2}=\left\{t_{0}\right\} \times\{0\} \times S_{3}^{1} \times\{0\} \times S_{6}^{1} \times\{0\} \times \mathbb{R}_{8}^{\geq 0} \times\left\{x_{0}\right\}
$$

and creates a Wilson-'t Hooft line of the type considered in section 3. It corresponds to a Verlinde operator in Toda theory on $C_{1, n}$, constructed from a vertex operator transported along the path $S_{6}^{1} \times\left\{\theta_{0}\right\}$. We will explain in a moment how to get the other relevant Verlinde operators.

To understand the relation to quantum integrable systems, let us compactify $\mathbb{R}_{9}$ to a circle $S_{9}^{1}$ of radius $R_{9}$. By doing so, we are uplifting the four-dimensional gauge theory to a five-dimensional one, compactified on a circle. Indeed, by T-duality on $S_{9}^{1}$ we get D5-branes Ď5 $5_{i}$, NS5-branes Nك̌5 ${ }^{r}$ and a D3-brane Ď3 with worldvolumes

$$
\begin{aligned}
M_{\check{\mathrm{D} 5} \text { }} & =\mathbb{R}_{0} \times \mathbb{R}_{12}^{2} \times{ }_{\epsilon} S_{3}^{1} \times\{0\} \times S_{6}^{1} \times\{0\} \times\{0\} \times \check{S}_{9}^{1}, \\
M_{\mathrm{NS}^{r}{ }^{r}} & =\mathbb{R}_{0} \times \mathbb{R}_{12}^{2} \times{ }_{\epsilon} S_{3}^{1} \times\{0\} \times\left\{l^{r}\right\} \times\{0\} \times \mathbb{R}_{8} \times \check{S}_{9}^{1}, \\
M_{\check{\mathrm{D} 3}} & =\left\{t_{0}\right\} \times\{0\} \times S_{3}^{1} \times\{0\} \times S_{6}^{1} \times\{0\} \times \mathbb{R}_{8}^{\geq 0} \times \check{S}_{9}^{1} .
\end{aligned}
$$

The D5- and NS5-branes intersect along $\mathbb{R}_{0} \times \mathbb{R}_{12}^{2} \times_{\epsilon} S_{3}^{1} \times \check{S}_{9}^{1}$, where a five-dimensional circular quiver theory arises. Recall that the radius $\check{R}_{9}$ of the dual circle $\breve{S}_{9}^{1}$ is inversely proportional to the original radius, $\check{R}_{9}=\alpha^{\prime} / R_{9}$.

Going back to the M-theory setup, we reduce it on $S_{3}^{1}$ and apply T-duality on $S_{9}^{1}$. Then, $\mathrm{M} 55_{i}$ become D5-branes $\widetilde{\mathrm{D} 5}{ }_{i}$ on

$$
M_{\widetilde{\mathrm{D} 5_{i}}}=\mathbb{R}_{0} \times \mathbb{R}_{12}^{2} \times\{0\} \times S_{6}^{1} \times\{0\} \times\{0\} \times \check{S}_{9}^{1} \times S_{10}^{1},
$$

$\mathrm{M} 5^{r}$ become D3-branes $\widetilde{\mathrm{D}}^{r}$ on

$$
M_{\widetilde{\mathrm{D}}^{r}}=\mathbb{R}_{0} \times \mathbb{R}_{12}^{2} \times\{0\} \times\left\{l^{r}\right\} \times\{0\} \times \mathbb{R}_{8} \times\left\{\phi^{r}\right\} \times\left\{\theta^{r}\right\},
$$

and M2 becomes a fundamental string $\widetilde{\mathrm{F} 1}$ on

$$
M_{\widetilde{\mathrm{F}} 1}=\left\{t_{0}\right\} \times\{0\} \times\{0\} \times S_{6}^{1} \times\{0\} \times \mathbb{R}_{8}^{\geq 0} \times\left\{\phi_{0}\right\} \times\left\{\theta_{0}\right\} .
$$

The $N$ D5-branes $\widetilde{\mathrm{D}}_{i}$ support $\mathcal{N}=(1,1)$ super Yang-Mills theory with gauge group $\mathrm{SU}(N)$ on $\mathbb{R}_{0} \times \mathbb{R}_{12}^{2} \times S_{6}^{1} \times \check{S}_{9}^{1} \times S_{10}^{1}$. This theory is, however, deformed because the product between $S_{3}^{1}$ and $\mathbb{R}_{12}^{2} \times \mathbb{R}_{45}^{2}$ was twisted, which induces nontrivial background fields after reduction on $S_{3}^{1}$. This deformation is of the type studied in [39] and the same as the one applied to the D5-brane theory in [19]. ${ }^{7}$ If we consider the reduction to fourdimensional $\mathcal{N}=4$ super Yang-Mills theory, this deformation descends to the S-dual of the $\Omega$-deformation [41].

\footnotetext{
${ }^{7}$ The equivalence to the construction of [19] can be seen as follows. Let us reduce the M-theory setup instead on $S_{10}^{1}$ and perform T-duality on $S_{9}^{1}$. Then, we obtain a type IIB setup whose geometry contains a twisted product between $\mathbb{R}_{12}^{2} \times \mathbb{R}_{45}^{2}$ and the torus $S_{3}^{1} \times \check{S}_{9}^{1}$. This is the starting point of the construction of [19]. The string background proposed in [19] is achieved by a sequence of dualities applied to this setup: T-duality on $S_{3}^{1} \times \check{S}_{9}^{1}$, then S-duality, and finally T-duality on the dual torus $\check{S}_{3}^{1} \times S_{9}^{1}$. The first T-duality realizes the $\Omega$-deformation [40], hence we are considering the $\mathrm{S}$-dual $\Omega$-deformation here. The point is
} 
In the sector in which the relevant supersymmetry is preserved, the deformation leads to localization of the path integral. As a result, this sector of the deformed theory is equivalent to a bosonic theory which, roughly speaking, may be understood as living at the origin of $\mathbb{R}_{12}^{2}$. This theory turns out to be a four-dimensional variant of Chern-Simons theory [19], with Planck constant $\hbar \propto \epsilon$. Four-dimensional Chern-Simons theory, here placed on $\mathbb{R}_{0} \times S_{6}^{1} \times \check{S}_{9}^{1} \times S_{10}^{1}$, depends topologically on the cylinder

$$
\Sigma=\mathbb{R}_{0} \times S_{6}^{1}
$$

and holomorphically on the torus

$$
E=\check{S}_{9}^{1} \times S_{10}^{1} .
$$

The D3-branes $\widetilde{\mathrm{D}}^{r}$ create line defects extending in the longitudinal direction of $\Sigma$ and located at the points

$$
w^{r}=\phi^{r}+\mathrm{i} \theta^{r}
$$

on $E$. The fundamental string $\widetilde{\mathrm{F} 1}$, on the other hand, creates a Wilson line in the vector representation that winds around the circumferential direction and is located at

$$
z_{0}=\phi_{0}+\mathrm{i} \theta_{0}
$$

on $E$. Thus, on the cylinder $\Sigma$, we have the same situation as in figure $1(\mathrm{c})$, in which a quantum spin chain was described in terms of lines on a cylinder.

Indeed, a quantum integrable system emerges from such a configuration of line operators in four-dimensional Chern-Simons theory [17]. The Hilbert space of the integrable system is the space of states of the field theory on a time slice (where the $x^{0}$-coordinate is constant) intersected by line operators extending in the time direction. On this Hilbert space act transfer matrices, which are Wilson lines in the $x^{6}$-direction. The integrability is a consequence of the topological-holomorphic nature of the theory: by the topological invariance on $\Sigma$, one can slide line operators winding around the cylinder continuously along the longitudinal direction; and if two such line operators are located at different points on $E$, one can move them past each other without encountering a phase transition, thereby establishing the commutativity of transfer matrices.

It was argued in [19], based on the earlier work [2, 3], that a crossing of line defects created by a D3-brane and a fundamental string produces the elliptic L-operator (2.21) with $z=z_{0}, w=w^{r}$ and $y=0^{8}$ (up to shifts by constants). The parameter $\tau$ is the

that S-duality is geometrized via M-theory as a "9-11 flip." In the above construction, the S-duality can be replaced by T-duality on $\check{S}_{3}^{1}$, a lift to M-theory and reduction on $S_{3}^{1}$, and T-duality on the M-theory circle $S_{10}^{1}$. The configuration right after the lift to M-theory is nothing but our original M-theory setup. The subsequent steps in the 9-11 flip differ from what we did to that setup only in the choice of the circle on which we perform T-duality, $S_{10}^{1}$ now whereas $S_{9}^{1}$ before. However, we are still supposed to perform T-duality on $S_{9}^{1} \times \breve{S}_{10}^{1}$ as the last step in the construction of [19]. (Note that the 9-11 flip has exchanged $S_{3}^{1}$ and $S_{10}^{1}$.) This fixes the discrepancy.

${ }^{8}$ More generally, D3 ${ }^{r}$ can be split into two semi-infinite D3-branes D3 $3_{+}^{r}$ and D3 ${ }_{-}^{r}$, each ending on the stack of D5-branes at $x^{8}=0$. The parameter $y$ is given by the separation of these two halves in $E$. In the five-dimensional circular quiver theory, the separation is proportional to the complex mass parameter $m^{r}$ for the bifundamental hypermultiplet charged under the $r$ th and $(r+1)$ th gauge groups. 


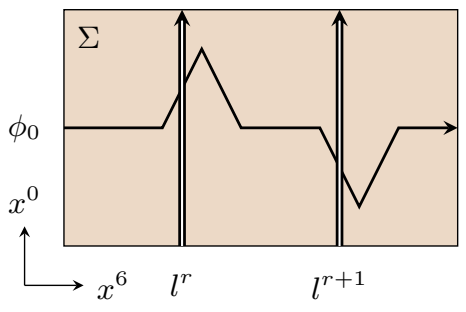

(a)

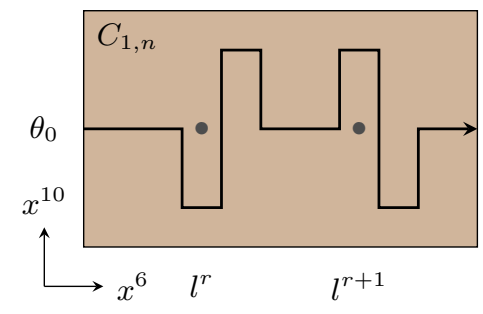

(b)

Figure 3. (a) A path in $\Sigma$ bending near double lines. (b) The corresponding path in $C_{1, n}$ detours around the punctures.

modulus of $E$ :

$$
\tau=\mathrm{i} \frac{R_{10}}{\check{R}_{9}} .
$$

Now, take the limit $\check{R}_{9} \rightarrow 0$, in which $\check{S}_{9}^{1}$ shrinks to a point, $S_{9}^{1}$ decompactifies, and the five-dimensional circular quiver theory reduces to the four-dimensional one. This is the trigonometric limit $\tau \rightarrow \mathrm{i} \infty$, so we conclude that the transfer matrix constructed from the trigonometric L-operator arises from a Wilson-'t Hooft line in the four-dimensional circular quiver theory.

In the previous sections we studied the transfer matrix $\mathcal{T}_{\sigma, m}$ associated with the cycle $\gamma_{\sigma}$ in $C_{1, n}$ specified by an $n$-tuples of signs $\sigma$. The Wilson-'t Hooft line considered above corresponds to a specific choice of $\sigma$. Those corresponding to the other choices can also be constructed in a similar manner, but the construction is a little more subtle. Let us explain how this construction works from the point of view of four-dimensional Chern-Simons theory.

For simplicity, let us set all $\theta^{r}=\theta_{0}$. (Since $\mathcal{T}_{\sigma, m}$ is independent of the spectral parameters $z_{0}$ and $w^{r}$, we do not lose anything by this specialization.) According to the analysis of [18], framing anomaly requires that if a Wilson line curves by an angle $\varphi$, its coordinate on $S_{10}^{1}$ must be shifted by $-\epsilon N \varphi / 2 \pi$. We can make use of this property to get a Wilson line supported on the cycle $\gamma_{\sigma}$ : fix a small value $\varphi_{0}$ and let the Wilson line bends by the angle $\sigma^{r} \varphi_{0}$ right before it crosses the $r$ th double line, as illustrated in figure 3 .

The trigonometric limit $\check{R}_{9} \rightarrow 0$ is equivalent to the limit $R_{10} \rightarrow \infty$. In this limit, $E$ is elongated by an infinite factor in the $x^{10}$-direction and the solid line is located at $z=\phi_{0}-\sigma^{r} \mathrm{i} \infty$ when it crosses the $r$ th double line. This is precisely the limit that appears in the definitions of the fundamental L-operators (2.38), from which $\mathcal{T}_{\sigma, m}$ is constructed.

Let us summarize the relation between the circular quiver theory and four-dimensional Chern-Simons theory. The circular quiver theory with gauge group $\mathrm{SU}(N)^{n}$ arises from six-dimensional $\mathcal{N}=(2,0)$ superconformal field theory of type $A_{N-1}$ compactified on an $n$-punctured torus, which is realized by a stack of $N$ M5-branes intersected by $n$ M5-branes. If we place this brane system in a twisted geometry, then by string dualities it is mapped to $N$ D5-branes and $n$ D3-branes in a particular string background. The D5-branes support six-dimensional $\mathcal{N}=(1,1)$ super Yang-Mills theory with gauge group $\mathrm{SU}(N)$, and this background deforms it. It is a topological-holomorphic sector of this deformed theory that is described by four-dimensional Chern-Simons theory. The D3-branes become line 
operators in four-dimensional Chern-Simons theory and prepare the Hilbert space of the integrable system. Finally, we can include M2-branes that create Wilson-'t Hooft lines in the circular quiver theory. They are mapped to fundamental strings in the dual frame and appear as Wilson lines in four-dimensional Chern-Simons theory. These Wilson lines act on the Hilbert space of the integrable system by transfer matrices.

Lastly, we remark that the D5-NS5-D3 brane system (5.9)-(5.11) is another interesting duality frame. It is actually possible to introduce an additional set of NS5-branes so that the 5-brane system realizes a four-dimensional $\mathcal{N}=1$ supersymmetric gauge theory on $\mathbb{R}_{12}^{2} \times{ }_{\epsilon} S_{3}^{1} \times \check{S}_{9}^{1}$. The D3-brane creates a surface defect in this theory. As expected, it acts on the partition function of the theory as an elliptic transfer matrix [2, 3].

\section{Acknowledgments}

We would like to thank Takuya Okuda, Masato Taki and Yutaka Yoshida for helpful discussions. KM is grateful to Perimeter Institute for hospitality. The work of KM is supported in part by JSPS KAKENHI Grant Number JP17K14296 and JP20K03935. TO is supported in part by JSPS KAKENHI Grant Number JP20J10126 and by RIKEN Junior Research Associate Program. The research of JY is supported by the Perimeter Institute for Theoretical Physics. Research at Perimeter Institute is supported by the Government of Canada through the Department of Innovation, Science and Economic Development and by the Province of Ontario through the Ministry of Research, Innovation and Science.

Open Access. This article is distributed under the terms of the Creative Commons Attribution License (CC-BY 4.0), which permits any use, distribution and reproduction in any medium, provided the original author(s) and source are credited.

\section{References}

[1] M. Bullimore, M. Fluder, L. Hollands and P. Richmond, The superconformal index and an elliptic algebra of surface defects, JHEP 10 (2014) 062 [arXiv:1401.3379] [INSPIRE].

[2] K. Maruyoshi and J. Yagi, Surface defects as transfer matrices, PTEP 2016 (2016) 113B01 [arXiv: 1606.01041] [INSPIRE].

[3] J. Yagi, Surface defects and elliptic quantum groups, JHEP 06 (2017) 013 [arXiv: 1701.05562] [INSPIRE].

[4] A. Kapustin, Wilson-'t Hooft operators in four-dimensional gauge theories and S-duality, Phys. Rev. D 74 (2006) 025005 [hep-th/0501015] [INSPIRE].

[5] K. Hasegawa, Ruijsenaars' commuting difference operators as commuting transfer matrices, Comm. Math. Phys. 187 (1997) 289.

[6] R.J. Baxter, Eight vertex model in lattice statistics and one-dimensional anisotropic Heisenberg chain. 2. Equivalence to a generalized ice-type lattice model, Annals Phys. 76 (1973) 25 [INSPIRE].

[7] M. Jimbo, T. Miwa and M. Okado, Solvable lattice models whose states are dominant integral weights of $A_{n-1}^{(1)}$, Lett. Math. Phys. 14 (1987) 123. 
[8] M. Jimbo, T. Miwa and M. Okado, Local state probabilities of solvable lattice models: an $A_{n-1}^{(1)}$ family, Nucl. Phys. B 300 (1988) 74 [INSPIRE].

[9] V. Pestun et al., Localization techniques in quantum field theories, J. Phys. A 50 (2017) 440301 [arXiv: 1608.02952] [INSPIRE].

[10] Y. Ito, T. Okuda and M. Taki, Line operators on $S^{1} \times R^{3}$ and quantization of the Hitchin moduli space, JHEP 04 (2012) 010 [Erratum ibid. 03 (2016) 085] [arXiv:1111.4221] [INSPIRE].

[11] L.F. Alday, D. Gaiotto and Y. Tachikawa, Liouville correlation functions from four-dimensional gauge theories, Lett. Math. Phys. 91 (2010) 167 [arXiv:0906.3219] [INSPIRE].

[12] N. Wyllard, $A_{N-1}$ conformal Toda field theory correlation functions from conformal $N=2$ $\mathrm{SU}(N)$ quiver gauge theories, JHEP 11 (2009) 002 [arXiv:0907.2189] [INSPIRE].

[13] L.F. Alday, D. Gaiotto, S. Gukov, Y. Tachikawa and H. Verlinde, Loop and surface operators in $N=2$ gauge theory and Liouville modular geometry, JHEP 01 (2010) 113 [arXiv: 0909.0945] [INSPIRE].

[14] N. Drukker, J. Gomis, T. Okuda and J. Teschner, Gauge theory loop operators and Liouville theory, JHEP 02 (2010) 057 [arXiv: 0909.1105] [INSPIRE].

[15] J. Gomis and B. Le Floch, 't Hooft operators in gauge theory from Toda CFT, JHEP 11 (2011) 114 [arXiv: 1008.4139] [INSPIRE].

[16] E.P. Verlinde, Fusion rules and modular transformations in $2 D$ conformal field theory, Nucl. Phys. B 300 (1988) 360 [inSPIRE].

[17] K. Costello, Supersymmetric gauge theory and the Yangian, arXiv:1303.2632 [INSPIRE].

[18] K. Costello, E. Witten and M. Yamazaki, Gauge theory and integrability, I, ICCM Not. 06 (2018) 46 [arXiv: 1709.09993] [INSPIRE].

[19] K. Costello and J. Yagi, Unification of integrability in supersymmetric gauge theories, arXiv: 1810.01970 [INSPIRE].

[20] G. Felder, Elliptic quantum groups, in $11^{\text {th }}$ international conference on mathematical physics (ICMP-11) (satellite colloquia: new problems in the general theory of fields and particles, Paris, France, 25-28 July 1994, pg. 211 [hep-th/9412207] [INSPIRE].

[21] G. Felder, Conformal field theory and integrable systems associated to elliptic curves, in Proceedings of the international congress of mathematicians, Birkhäuser, Basel, Switzerland (1995), pg. 1247 [hep-th/9407154] [INSPIRE].

[22] P. Etingof and A. Varchenko, Solutions of the quantum dynamical Yang-Baxter equation and dynamical quantum groups, Commun. Math. Phys. 196 (1998) 591 [q-alg/9708015].

[23] J.-L. Gervais and A. Neveu, Novel triangle relation and absence of tachyons in Liouville string field theory, Nucl. Phys. B 238 (1984) 125 [INSPIRE].

[24] R.J. Baxter, Eight-vertex model in lattice statistics, Phys. Rev. Lett. 26 (1971) 832 [INSPIRE].

[25] R.J. Baxter, Partition function of the eight vertex lattice model, Annals Phys. 70 (1972) 193 [INSPIRE].

[26] A.A. Belavin, Dynamical symmetry of integrable quantum systems, Nucl. Phys. B 180 (1981) 189 [INSPIRE]. 
[27] V.V. Bazhanov and S.M. Sergeev, A master solution of the quantum Yang-Baxter equation and classical discrete integrable equations, Adv. Theor. Math. Phys. 16 (2012) 65 [arXiv: 1006.0651] [INSPIRE].

[28] V.V. Bazhanov and S.M. Sergeev, Elliptic gamma-function and multi-spin solutions of the Yang-Baxter equation, Nucl. Phys. B 856 (2012) 475 [arXiv:1106.5874] [inSPIRE].

[29] D. Gaiotto, G.W. Moore and A. Neitzke, Four-dimensional wall-crossing via three-dimensional field theory, Commun. Math. Phys. 299 (2010) 163 [arXiv:0807.4723] [INSPIRE].

[30] T.D. Brennan and G.W. Moore, Index-like theorems from line defect vevs, JHEP 09 (2019) 073 [arXiv: 1903.08172] [INSPIRE].

[31] T.D. Brennan, A. Dey and G.W. Moore, On 't Hooft defects, monopole bubbling and supersymmetric quantum mechanics, JHEP 09 (2018) 014 [arXiv:1801.01986] [INSPIRE].

[32] T.D. Brennan, Monopole bubbling via string theory, JHEP 11 (2018) 126 [arXiv: 1806.00024] [INSPIRE].

[33] T.D. Brennan, A. Dey and G.W. Moore, 't Hooft defects and wall crossing in SQM, JHEP 10 (2019) 173 [arXiv: 1810.07191] [INSPIRE].

[34] B. Assel and A. Sciarappa, On monopole bubbling contributions to 't Hooft loops, JHEP 05 (2019) 180 [arXiv:1903.00376] [INSPIRE].

[35] S.N.M. Ruijsenaars, Complete integrability of relativistic Calogero-Moser systems and elliptic function identities, Commun. Math. Phys. 110 (1987) 191 [INSPIRE].

[36] D. Gaiotto, $N=2$ dualities, JHEP 08 (2012) 034 [arXiv:0904.2715] [InSPIRE].

[37] D. Gaiotto, G.W. Moore and A. Neitzke, Wall-crossing, Hitchin systems, and the WKB approximation, arXiv:0907.3987 [INSPIRE].

[38] E. Witten, Solutions of four-dimensional field theories via M-theory, Nucl. Phys. B 500 (1997) 3 [hep-th/9703166] [INSPIRE].

[39] J. Yagi, $\Omega$-deformation and quantization, JHEP 08 (2014) 112 [arXiv:1405.6714] [INSPIRE].

[40] S. Hellerman, D. Orlando and S. Reffert, String theory of the $\Omega$ deformation, JHEP 01 (2012) 148 [arXiv:1106.0279] [INSPIRE].

[41] N.A. Nekrasov, Seiberg-Witten prepotential from instanton counting, Adv. Theor. Math. Phys. 7 (2003) 831 [hep-th/0206161] [INSPIRE]. 